\title{
ESTIMATING MARINE RESERVOIR EFFECTS IN ARCHAEOLOGICAL CHRONOLOGIES: COMPARING $\triangle$ R CALCULATIONS IN PRINCE RUPERT HARBOUR, BRITISH COLUMBIA, CANADA
}

\author{
Andrew Martindale, Gordon T. Cook, Iain McKechnie, Kevan Edinborough, Ian Hutchinson, \\ Morley Eldridge, Kisha Supernant, and Kenneth M. Ames
}

\begin{abstract}
The best method for quantifying the marine reservoir effect (MRE) using the global IntCal Marine 13 calibration curve remains unresolved. Archaeologists frequently quantify uncertainty on MRE values as errors computed from single pairs of marineterrestrial radiocarbon ages, which we argue significantly overstates their accuracy and precision. Here, we review the assumptions, methods, and applications of estimating MRE via an estimate of the additional regional offset between the marine and terrestrial calibration curves ( $\triangle R$ ) for the Prince Rupert Harbour (PRH) region of British Columbia, Canada. We acknowledge the influence on $\triangle R$ of MRE variation as (1) a dynamic oceanographic process, (2) its variable expression in biochemical and geochemical pathways, and (3) compounding errors in sample selection, measurement, and calculation. We examine a large set of marine-terrestrial pairs $(\mathrm{n}=63)$ from PRH to compare a common archaeological practice of estimating uncertainty from means that generate an uncertainty value of \pm 49 years with a revised, more appropriate estimate of error of \pm 230 years. However, we argue that the use of multiple-pair samples estimates the $P R H \Delta R$ as $273 \pm 38$ years for the last 5,000 years. Calculations of error that do not consider these issues may generate inaccurate age estimates with unjustifiable precision.
\end{abstract}

El mejor método para cuantificar el efecto reservorio marino (MRE, por sus siglas en inglés) usando la curva global de calibración IntCal Marine 13 permanece sin resolver. Los arqueólogos frecuentemente cuantifican la incertidumbre en valores del MRE como errores calculados a partir de pares únicos de edades radiocarbónicas marinas y terrestres que, sostenemos, sobrevaloran significativamente su exactitud y precisión. Aquí revisamos las suposiciones, métodos y aplicaciones para estimar el MRE a través de una estimación de la compensación regional adicional entre las curvas de calibración marinas y terrestres $(\Delta R)$ para la región de Prince Rupert Harbour (PRH) en Columbia Británica, Canadá. Reconocemos la influencia sobre el $\Delta R$ de variaciones del MRE como (1) un proceso oceanográfico dinámico, (2) su expresión variable en caminos bioquímicos y geoquímicos que producen muestras para datación ${ }^{14} C$ por AMS y (3) errores compuestos en la selección de muestras, mediciones y cálculos. Examinamos un amplio conjunto de pares marinos-terrestres $(n=63)$ procedentes de PRH para comparar la práctica arqueológica común de estimar la incertidumbre a partir de promedios que generan un valor de incertidumbre de \pm 49 años, con una estimación de error revisada, más apropiada, de \pm 230 años. Este acercamiento estima el $\Delta R$ de PRH en $273 \pm 38$ años para los últimos 5,000 años. Los cálculos de error que no consideran estas cuestiones pueden generar estimaciones inexactas de edad con precisiones injustificables.

\footnotetext{
Andrew Martindale — Department of Anthropology, University of British Columbia, 6303 N.W. Marine Drive, Vancouver, British Columbia V6 T 1Z1, Canada (andrew.martindale@ubc.ca, corresponding author)

Gordon T. Cook $\square$ Scottish Universities Environmental Research Centre Radiocarbon Dating Laboratory, Scottish Enterprise Technology Park, Rankine Avenue, East Kilbride G75 0QF, UK

Iain McKechnie - Department of Anthropology, University of Victoria, Cornett Building, Room B246a, P.O. Box 1700, STN CSC, Victoria, British Columbia V8W 2Y2, Canada; and Hakai Institute, P.O. Box 309, Heriot Bay, British Columbia V0P 1H0, Canada

Kevan Edinborough — University College London, 31-34 Gordon Square, London WC1H OPY, UK

Ian Hutchinson — Department of Geography, 8888 University Drive, Simon Fraser University, Burnaby, British Columbia V5A 1S6, Canada

Morley Eldridge - Millennia Research Limited, 501 Alpha Street, Victoria, British Columbia V8Z 1B2, Canada

Kisha Supernant $\square$ Department of Anthropology, University of Alberta, 13-15 Tory Building, Edmonton, Alberta T6 G 2H4, Canada

Kenneth M. Ames a Department of Anthropology, Portland State University, Portland, OR 97207, USA
}

American Antiquity 83(4), 2018, pp. 659-680

Copyright (C) 2018 by the Society for American Archaeology

doi:10.1017/aaq.2018.47 
$\mathrm{R}$ adiocarbon $\left({ }^{14} \mathrm{C}\right)$ dating of marinesourced carbon samples has considerable value in coastal archaeology and represents a significant percentage of the cumulative global radiocarbon record in coastal settings. However, its effective use is hampered by complexities of carbon pathways in marine systems, changes in such systems over time, and ongoing debates about the most appropriate way to sample and quantify the difference between them. Debates within geologic and geochemical literature are foundational to the archaeological purpose of estimating ages and rates of processes. We demonstrate that common archaeological calibration practices underestimate uncertainty, especially compared with revised estimates of error proposed by Cook and colleagues (2015). We show that increasing the sample of data points of the difference between marine and terrestrial systems does not necessarily increase either the accuracy (the proximity of results to reality) or the precision (the range of uncertainty in results) of such estimations. The main culprit for the disjunction between sample size and accuracy is likely the mismatching of marineterrestrial pairs from different chronological contexts. We also note a consistent and pervasive overstatement of precision in archaeological estimates, largely due to inappropriate calculations of error. Using a large set $(n=63$, though 48 of these pairs were derived from 24 dates; see below) of marine-terrestrial pairings from the Prince Rupert Harbour region of British Columbia, Canada, we demonstrate that the most accurate, precise, and cost-effective method for correcting marine-sourced radiocarbon samples is the multiple-pair sample approach developed by Ascough and colleagues (2007), Russell and colleagues (2011), and Cook and colleagues (2015). We see value in wider application of this method in coastal archaeology.

Kintigh (2015) notes that many computational presentations of archaeological mathematics gloss over both the logical implications and the mathematical steps. As with any academic genre, such an approach is efficient when directed at disciplinary peers but generates exclusion of those not familiar with the foundational ideas or the applied methods. Kintigh writes,
If practicing archaeologists lack reasonable access to a method, it is unlikely to achieve widespread use. I here use "access" in two senses, that the method can be adequately understood and that it is possible to find and use the software tools to execute it [2015:488].

Given its wide use and value, radiocarbon dating in general, and its application to marine-sourced carbon in particular, is explored by many archaeologists who do not have much experience with quantitative methods. Thus, we see value in both an assessment of logical and mathematical options and a step-by-step guide to best practices (including supplemental Microsoft Excel spreadsheet templates for conducting the calculations), which we elaborate on below.

The calibration of radiocarbon ages, estimated from samples containing marine-derived carbon, is complicated by a spatially and temporally variable reservoir effect that makes these samples appear too old (Mangerud and Gulliksen 1975; Stuiver et al. 1986). Radiocarbon is produced in the stratosphere, rapidly oxidizes to carbon dioxide, and diffuses through the troposphere and into terrestrial biological systems via organic carbon production (fixing) through photosynthesis. The timescale for these biogeochemical processes is short, on the order of 10 to 20 years (Ascough et al. 2004). This creates a correlation between the annual production of radiocarbon and its accumulation in the terrestrial organic carbon reservoir that requires calibration via the IntCal 13 curve to correct for variation in ${ }^{14} \mathrm{C}$ production (Reimer et al. 2013; Stuiver et al. 1986). Because of the slow rate of diffusion of carbon dioxide into water and the isolation of bodies of water from the ocean/atmosphere interface for potentially many hundreds of years, marine biochemical pathways effectively fix carbon from older carbon sources than terrestrial systems, with correspondingly depleted levels of radiocarbon. Hence, marine samples generate artificially older age values. The resulting difference between the terrestrial and marine systems is expressed as a cumulative difference between the terrestrial and marine calibration curves for radiocarbon ages, known as the marine reservoir effect (MRE). 
The MRE differs through the ocean water column, but most archaeological samples derive from the surface seawater (mixed) photic zone, where photosynthetic uptake of inorganic carbon occurs (Ascough et al. 2004:611). MRE is expressed mathematically as $\mathrm{R}(\mathrm{t})=R+\Delta \mathrm{R}$, or the sum of the global difference $(\mathrm{R})$ and the additional local value that modifies it $(\Delta R)$. Although MRE calculations and estimates of both $R$ and various $\Delta R$ values have been explored for decades, the process of enumerating and calibrating the cumulative effects of marine/ terrestrial differences is undergoing continual refinement (see Hutchinson et al. 2004 for a discussion of this).

The current global value of $\mathrm{R}$, at $\sim 400$ years, is a modeled estimate of the average age difference between surface marine and terrestrial carbon reservoirs (Hughen et al. 2004). Early enumerations of $\Delta \mathrm{R}$ were based on the radiocarbon dating of modern pre-atomic bomb marine samples (usually shells of intertidal marine invertebrates) of known age and location, to estimate $\Delta \mathrm{R}$ into antiquity. Stuiver and colleagues (1986) defined a $\Delta \mathrm{R}$ intercept estimate based on the difference between the conventional marine radiocarbon age and the equivalent marine age projected from the intercept of a stratigraphically (and thus chronologically) paired terrestrial age (see Stuiver and Braziunas 1993:153 for calculating the intercept value; see Russell et al. 2011: Figure 1 for a graphic illustration of this logic).

Increasingly, $\Delta \mathrm{R}$ is calculated using a sample of marine carbon for which the terrestrial/atmospheric ${ }^{14} \mathrm{C}$ age is known; that is, it is stratigraphically paired, usually from an archaeological context, to a ${ }^{14} \mathrm{C}$-dated terrestrial sample with the assumption that deposition was coeval. The terrestrial/atmospheric ${ }^{14} \mathrm{C}$ age $\pm 1 \sigma$ of the terrestrial carbon sample is converted to a modeled marine ${ }^{14} \mathrm{C}$ age via interpolation between the IntCal13 atmospheric curve and the Marine13 curve (Reimer et al. 2013). $\Delta \mathrm{R}$ is simply the difference between this modeled marine ${ }^{14} \mathrm{C}$ age and the conventional ${ }^{14} \mathrm{C}$ age of the marine carbon sample. The $1 \sigma$ error on this difference is calculated by the propagation of the errors on the individual measurements.

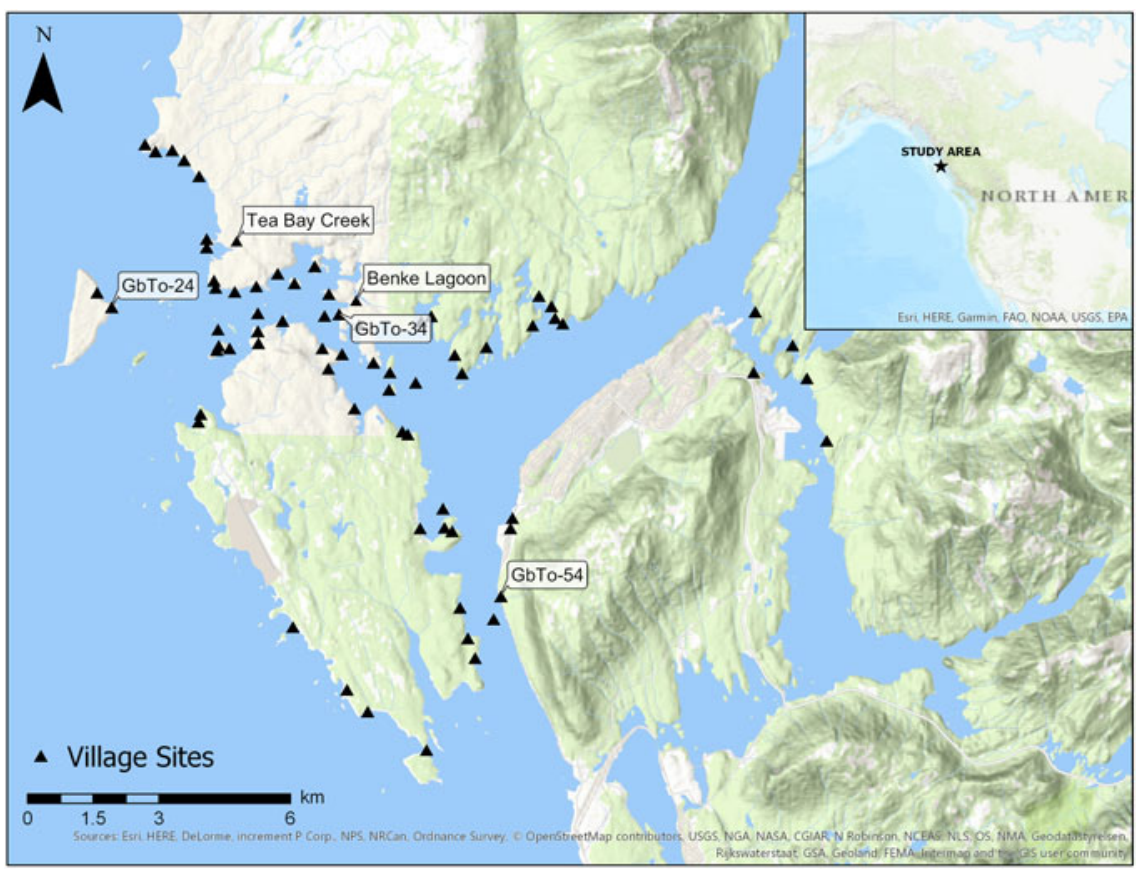

Figure 1. Map of the Prince Rupert Harbour study area showing radiocarbon-dated site locations. Labels indicate sites that have paired marine-terrestrial $\Delta R$ estimates shown in Figure 2. (Color online) 


\section{Sources of Uncertainty in Marine Reservoir Effects}

As Stuiver and colleagues (1986:980) observe in their foundational analysis, the first approximation of $\Delta \mathrm{R}$ as a constant value must be assumed in the absence of a representative sample of MRE data over time. It is important to note, following Stuiver and colleagues (1986:986) and Stuiver and Braziunas (1993:139), that while $\mathrm{R}(\mathrm{t})$ varies over time, $\Delta \mathrm{R}$ does not vary if local conditions remain stable enough to create a constant difference between the marine and terrestrial curves (cf. Weisler et al. 2017). By this preliminary logic, a suitably derived $\Delta \mathrm{R}$ from any time period serves as a proxy for all time periods. However, MRE processes are potentially highly variable over short distances and have the potential to shift over time, especially in the context of global postglacial environmental changes affecting oceanographic circulation, rendering $\Delta \mathrm{R}$ calculations susceptible to dramatic variation (e.g., Gómez et al. 2008; Goodfriend and Flessa 1997; Heier-Nielsen et al. 1995; Ingram and Southon 1996; Kennett et al. 1997; Kovanen and Easterbrook 2002; Rick et al. 2012; Southon and Fedje 2003; Southon et al. 1990; Stuiver and Braziunas 1993; Stuiver et al. 1986; Taylor et al. 2007). As Misarti and colleagues (2009) and Spzak and colleagues (2018) illustrate for the North Pacific and Bering Sea, there is a high probability of a major environmental change occurring in the northern oceans between the modern and ancient periods that likely influenced the reservoir age, making this approach less applicable to archaeological chronologies. Variability in $\Delta \mathrm{R}$ values across space and time undermines the use of marinesourced carbon for both relative and absolute chronological estimates, a significant issue for coastal archaeology (Ascough et al. 2004). Hutchinson and colleagues (2004) argue that in most coastal contexts, stability in MRE conditions over time is unlikely, creating an empirical problem to be tested. However, both Hua and colleagues (2015) and Weisler and colleagues (2009) present examples of stable $\Delta R$ periods in the context of changes over time and consistency over regions, respectively, reminding us that patterns of change or stability are possible but need demonstration in each local context.

While the extent of variability in MRE over time and space remains unclear for many archaeological contexts, the sources of variation are relatively well known. Hutchinson and colleagues (2004) anticipate variation both in the marine water column and in marine carbon pathways. The former may derive from changes in oceanographic and nearshore hydrologic conditions that mix deep ocean waters with nearsurface layers, while the latter include variation in the chemical and biochemical pathways through which carbon is fixed in marine tissues (Hutchinson et al. 2004; Rick et al. 2012; Stocker and Wright 1998; Stuiver and Braziunas 1993; Taylor et al. 2007). Hutchinson and colleagues (2004:194-195) also include carbon leaching at the outer surface of marine shell after death, variation in carbon uptake and feeding strategies by different marine invertebrates, and seasonal growth variation as examples (see also Petchey et al. 2008). Some of these variables can be controlled by sample selection for marine species with known or near-surface habitat ranges. However, these factors can render MRE too heterogeneous to be easily estimated in some contexts. For example, Etayo-Cadavid and colleagues (2013) note a pattern of considerable variation $(\sim 150-400$ years) in $\Delta \mathrm{R}$ variability from shortlived marine mollusks (Donax obesulus) in coastal Peru over the last 2,000 years that they associate with increased short-term variation in deepwater upwelling caused by El Niño events. Similar challenges are outlined in Deo and colleagues (2004) and Gómez and colleagues (2008), discussed below.

Recent scholarship identifies variation in $\Delta \mathrm{R}$ values from the same locations at different times, often from samples derived from archaeological contexts (Eldridge et al. 2014; Goodfriend and Flessa 1997; Ingram and Southon 1996; Kennett et al. 1997; McKechnie and Eldridge 2013; Stocker and Wright 1998; Stuiver and Braziunas 1993; Voelker et al. 1998), generating a chronological curve in some locations. Given the potential for a variable MRE regime across space and time, the challenge for archaeologists is to balance the ambitions of accuracy (the proximity of the $\Delta \mathrm{R}$ value to reality) and 


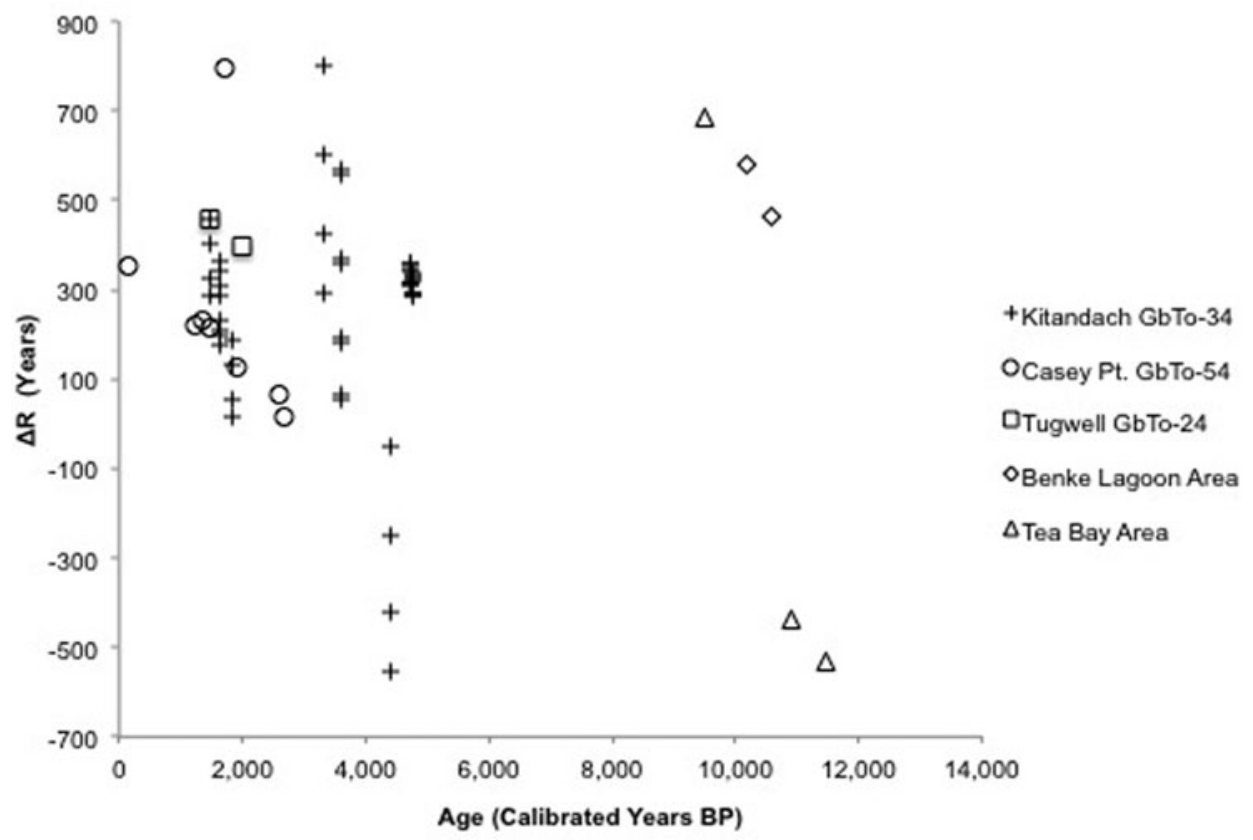

Figure 2. $\Delta \mathbf{R}$ values from all marine-terrestrial pairs in Prince Rupert Harbour by location. Note that the marineterrestrial pairs from the Benke Lagoon area and one sample from the Tea Bay area $(\Delta R=681$ years $)$ were selected from bulk samples. They are more likely to derive from mismatched pairs than the other samples presented in this figure. Similarly, the negative $\Delta \mathrm{R}$ values from Kitandach and Tea Bay are likely the result of mismatched pairs, as they suggest higher ${ }^{14} \mathrm{C}$ concentrations in the oceans than in the atmosphere.

precision (the uncertainty of the $\Delta \mathrm{R}$ calculation at different points in time/space). The cost of sample retrieval and ${ }^{14} \mathrm{C}$ dating often influences outcomes, with most archaeological programs using extant $\Delta \mathrm{R}$ estimates or single marineterrestrial pairs. Here we review the appropriate estimate of the MRE via the calculation of a local value for $\Delta \mathrm{R}$. We then turn to a case study in the Prince Rupert Harbour (PRH) area of north coastal British Columbia, Canada, to compare different approaches to calculating $\Delta \mathrm{R}$ and its uncertainty.

\section{Calculating $\Delta \mathbf{R}$}

The evaluation of MRE differs somewhat between archaeological and geochemical approaches. Geochemists tend to build temporally and spatially constrained datasets from which to assess $\Delta \mathrm{R}$ values, while archaeologists usually seek $\Delta \mathrm{R}$ values to estimate the correct age of previously dated samples. The archaeological application of $\Delta \mathrm{R}$ to existing ${ }^{14} \mathrm{C}$ datasets, often spanning large time periods and/or regions that are oceanographically diverse, promotes a circular logic in which the expectation that $\Delta \mathrm{R}$ is measurable and useful is assumed before evidence is collected. Consider the following geochemical (Gómez et al. 2008) and archaeological (Deo et al. 2004) examples.

Gómez and colleagues (2008) calculated $\Delta \mathrm{R}$ values from 18 pre-bomb, early twentiethcentury shell samples of known age from seven locations along the northeastern coast of Argentina. They identified high variation (143-2,482 years) in $\Delta \mathrm{R}$ between locations, likely the consequence of the dynamic and varied carbon cycles in local hydrologic and oceanographic effects, particularly the effects of large estuaries and variability in freshwater inputs in this coastal region. The authors conclude that the analytical resolution and hence interpretive value of marine shell in ${ }^{14} \mathrm{C}$ chronologies over the sampled time periods in this region is low. Gómez and colleagues do not calculate dispersion but argue that the variation in the region in all but one location is too great 
to assess $\Delta \mathrm{R}$ as a constant, concluding that building chronologies with marine-sourced carbon in the area is "seriously limited" (2008:128).

In contrast, archaeologists regularly seek $\Delta \mathrm{R}$ values for predetermined age ranges and regions, based on the existing use of ${ }^{14} \mathrm{C}$ data in chronology building. Indeed, in many contexts, imperfect $\Delta$ Rs are already applied, and the archaeological effort is to refine and correct existing conclusions. For example, Deo and colleagues (2004) used shell-charcoal pairs from previously excavated archaeological samples in Washington State to assess marine reservoir effects over the last 3,000 years in the Salish Sea, including the American Gulf Islands and Puget Sound. A $\Delta \mathrm{R}$ of $400 \pm 23$ years was widely employed, based on previously published regional estimates of modern, pre-bomb samples (Robinson and Thompson 1981). Deo and colleagues (2004) identified a fluctuating MRE based on the analysis of 18 marine-terrestrial pairs, which produced $\Delta \mathrm{R}$ estimates ranging from -504 to 2,281 years over the last 3,000 years. They propose $\Delta \mathrm{R}$ values of 0 (from 0 to $500{ }^{14} \mathrm{C}$ years $\mathrm{BP}$ ), -400 (from 500 to $1200 \mathrm{BP}$ ), and 0 (from 1200 to $3000 \mathrm{BP}$ ), ${ }^{1}$ while outliers were identified subjectively as values away from the "internal consistency" of the pattern (2004:779).

The differences between these examples are significant for archaeology, and each approach has considerable mathematical and logical implications. If MRE is constant over time and stable over wide regions, then $\Delta \mathrm{R}$ can be reliably estimated from small, spatially or temporally constrained samples, such as modern, pre-bomb invertebrate shells of known age. In contexts where this is supported, archaeologists can rely on existing regional estimates and need not dedicate funds or effort to resolving MRE via $\Delta \mathrm{R}$. However, stability cannot be assumed, and estimating $\Delta \mathrm{R}$ in variable contexts remains a logistical and financial challenge. Importantly, archaeological estimates of uncertainty in $\Delta \mathrm{R}$ tend to be either judgmental (e.g., identifying anomalies during exploratory data analysis) or calculated as simply the combination of the measurement errors on the marine and terrestrial radiocarbon age estimates. We argue that recent research supports more robust methods for estimating error in the association of marine and terrestrial samples.

\section{Outliers and Sample Selection}

Archaeologists are often reluctant to conclude that in some contexts, marine-sourced carbon cannot be effectively used in chronologies, assuming instead that additional paired samples will resolve variation and reduce uncertainty. This is essentially a debate about outliers and more broadly the interpretive utility and unresolved uncertainty of marine-influenced radiocarbon dates. Anticipation of consistent MRE conditions expects redundant samples from a single location to be unimodal and thus vary around a measure of central tendency. Deviations from such a pattern are interpreted as outliers whose influence can be minimized by trimming the dataset (for example, ignoring values beyond the interquartile spread), by differential weighting to the modality (for example, via a weighted mean), or by reducing the temporal or spatial range of the $\Delta \mathrm{R}$ assessment based on available data.

The expectation that MRE conditions change over time anticipates $\Delta \mathrm{R}$ as a curve that can be estimated as a sequential pattern of central tendencies over a series of times in a location or region. Here, outliers are identified against the curve, either as a central tendency calculated within temporal bins or as a polynomial trend line through the data. It is uncommon for archaeologists to collect a sufficient sample of marine-terrestrial pairs to mathematically identify outliers. It is more common, such as in Deo and colleagues (2004) and Eldridge and colleagues (2014), for a $\Delta \mathrm{R}$ curve and its outliers to be identified judgmentally.

As Deo and colleagues (2004:775) note, the archaeological context is challenged by potentially compounding sources of error. Archaeological assessments of $\Delta \mathrm{R}$ typically employ pairs of terrestrial- and marine-sourced carbon from the same stratigraphic context. The first, and perhaps most ubiquitous, error is the lack of securely contemporary samples derived from the same chronological context; variation here can add considerable uncertainty that will not be resolvable through subsequent measurement precision, a problem that cannot be overstated. A given terrestrial date has its own ${ }^{14} \mathrm{C}$ measurement error: an error that is not introduced when marine samples of known age are used, such as 
in Gómez and colleagues (2008). The contemporaneity (coeval deposition) of marine and terrestrial samples is also a source of uncertainty, as even spatial proximity does not preclude a difference in the year of death of marine and terrestrial organisms. Charcoal and shell are commonly dated materials, and both are susceptible to a time gap between the death of the organism and its deposition in archaeological sediments, the so-called old wood and old shell effects (e.g., Rick et al. 2005). However, this problem can be addressed with an additional line of dating evidence independent of the radiocarbon measurements or calibration curve, such as that yielded by uranium-thorium series dating (Burley et al. 2015; Weisler et al. 2017) or optically stimulated luminescence (Neudorf et al. 2017).

In the context of such variation, increasing sample size within narrow (in archaeological terms) temporal bins of 500 to 1,000 years may improve the accuracy and precision of $\Delta \mathrm{R}$ estimates. However, this is not necessarily the case, and events lasting as little as a few decades can introduce significant variability within 500to 1,000-year bins. The prospect of increasing sample sizes at such a temporal scale presents a major financial barrier to archaeologists and has no guarantee of a meaningful reduction in uncertainty. As we discuss below, the multiplepair approach developed by Ascough and colleagues (2007), Russell and colleagues (2011), and Cook and colleagues (2015) provides a useful balance between accuracy and cost by making the best use of the statistical properties of ${ }^{14} \mathrm{C}$ age measurements.

\section{Calculation of $\Delta R$ from a Single Marine-Terrestrial Pair}

A $\Delta \mathrm{R}$ result can be calculated in one of four ways. Stuiver and colleagues (1986) outline three ways: first, as the difference between the known age of a pre-bomb marine sample and its ${ }^{14} \mathrm{C}$ age; second, as the difference in ${ }^{14} \mathrm{C}$ years between paired marine and terrestrial samples, allowing the adjusted marine ${ }^{14} \mathrm{C}$ age to be calibrated via the terrestrial curve; and, third, as the difference between the conventional marine age and a modeled marine age, the latter being derived from the paired terrestrial sample. Stuiver and colleagues (1986) argue that the latter is preferred in contexts without historically known ages, and it has become the standard geochemical approach for archaeological samples (see also Russell et al. 2011:278-280).

Fourth, recently Reimer and Reimer (2017) announced an online tool (deltar: calib.org/ marine) for computing $\Delta \mathrm{R}$ from known-age, prebomb marine samples or contemporaneously paired marine and terrestrial samples. The deltar program computes $\Delta \mathrm{R}$ using the full calibrated probability distribution function rather than just the intercepts, making it a more accurate assessment of MRE.

Published estimates of $\Delta \mathrm{R}$, such as in the PRH region and elsewhere on the Northwest Coast (Table 1), are often inconsistent in the application of these approaches. The first approach is common in geochemical analyses of historically collected shells, such as McNeely and colleagues (2006), who report a range of $\Delta \mathrm{R}$ values for Haida Gwaii and southeast Alaska from 410 to 670 years, with measurement errors of $\pm 20-70$ years. Early archaeological efforts, published before the first marine calibration curve, Marine98 (Stuiver et al. 1998), employed the second approach (Archer 1992; Moss and Erlandson 1992; Southon et al. 1990), generating $\Delta \mathrm{R}$ estimates ranging from 245 to 430 years, with estimated errors of $\pm 50-100$ years. More recent work has relied on regional estimates (Ames 2005; Cybulski 2016; McLaren 2008) or employed the third approach (Edinborough et al. 2016; Eldridge et al. 2014; Martindale et al. 2009; McKechnie and Eldridge 2013).

Since archaeologists typically use either the second or third approach, the differences between these are usefully illustrated with an example. Southon and Fedje (2003:107) report the ${ }^{14} \mathrm{C}$ ages of stratigraphically paired charcoal (CAMS-49625, 1560 \pm 40 ) and marine shell (Saxidomus; CAMS-49626, 2370 \pm 50 ) from the PRH site of GbTo-24 (Table 2). They report the value of the difference between the means of the uncalibrated marine and terrestrial ages $(\mathrm{R}[\mathrm{t}]=810)$ with an error of \pm 60 years (though a simple combination of errors would return a value of \pm 64 ); thus (using an $R$ value of 405 years) the resulting $\Delta \mathrm{R}$ is $405 \pm 60$. Southon and Fedje are cautious not to report a specific 
Table 1. Regional Calculations of $\Delta \mathrm{R}$ in the Prince Rupert Harbour (PRH) Environs Listed by Date or Publication.

\begin{tabular}{|c|c|c|c|c|}
\hline Location & Source(s) & $\begin{array}{c}\Delta \mathrm{R} \\
\text { Estimates }^{\mathrm{a}}\end{array}$ & $\begin{array}{c}\text { Error } \\
\text { Estimate }\end{array}$ & Sample \\
\hline $\begin{array}{r}\text { Southeast } \\
\text { Alaska }\end{array}$ & Moss 1989:537 & 278 & \pm 50 & Regionally calculated mean \\
\hline Haida Gwaii & Southon et al. 1990 & 30 to 380 & \pm 100 & $\begin{array}{l}25 \text { shell-wood pairs from stratigraphically intact } \\
\text { archaeological and paleoecological deposits }\end{array}$ \\
\hline PRH & Archer 1992, 2001 & 245 & \pm 50 & $\begin{array}{l}\text { Estimate from regional data including three } \\
\text { shell-wood pairs: Pavlov Harbor, Alaska; FjUb-10 } \\
\text { FiTq-2 }\end{array}$ \\
\hline $\begin{array}{l}\text { North Pacific } \\
\text { coast }\end{array}$ & $\begin{array}{l}\text { Stuiver and Braziunas } \\
1993\end{array}$ & 395 & \pm 25 & $\begin{array}{l}\text { Estimated from published and compiled results of } \\
\text { modern marine samples }\end{array}$ \\
\hline Haida Gwaii & Southon and Fedje 2003 & $195^{\mathrm{b}}$ & \pm 100 to 200 & $\begin{array}{l}20 \text { shell-wood pairs from stratigraphically intact } \\
\text { archaeological and paleoecological deposits }\end{array}$ \\
\hline $\mathrm{PRH}$ & $\begin{array}{l}\text { Southon and Fedje } \\
\text { 2003:107 }\end{array}$ & 335 and 405 & \pm 60 and \pm 70 & Separate shell-charcoal pairs \\
\hline $\begin{array}{l}\text { North Pacific } \\
\text { coast }\end{array}$ & Ames 2005 & 395 & \pm 25 & Beta-Analytic \\
\hline $\begin{array}{r}\text { Southeast } \\
\text { Alaska }\end{array}$ & McNeely et al. 2006 & 410 to $670^{c}$ & \pm 20 to 70 & $\begin{array}{l}17 \text { live-collected shells from the early twentieth } \\
\text { century housed in museum collections }\end{array}$ \\
\hline Haida Gwaii & McNeely et al. 2006 & 200 to $390^{\mathrm{c}}$ & \pm 40 to 50 & Five live-collected shells from museum collections \\
\hline $\begin{array}{r}\text { Southeast } \\
\text { Alaska }\end{array}$ & Barron et al. 2009 & 330 to $385^{\mathrm{d}}$ & - & $\begin{array}{l}\text { Three shell-wood pairs from different (unspecified) } \\
\text { locations along coastal Alaska }\end{array}$ \\
\hline $\begin{array}{l}\text { Dundas } \\
\text { Islands }\end{array}$ & $\begin{array}{l}\text { McLaren 2008; } \\
\quad \text { McLaren et al. } 2011^{\mathrm{e}}\end{array}$ & 215 to 370 & \pm 30 to 60 & $\begin{array}{l}\text { Stratigraphically associated shell and charcoal from } \\
\text { percussion cores }\end{array}$ \\
\hline $\begin{array}{r}\text { Southeast } \\
\text { Alaska }\end{array}$ & $\begin{array}{l}\text { Carlson 2012; Carlson } \\
\text { and Baichtal } 2015\end{array}$ & 545 & \pm 60 & Four shell-wood pairs from Prince of Wales Island \\
\hline $\begin{array}{l}\text { PRH, Dundas } \\
\text { Islands }\end{array}$ & $\begin{array}{l}\text { McKechnie and } \\
\text { Eldridge } 2013\end{array}$ & 250 to 450 & \pm 60 & $\begin{array}{l}\text { Results published in McLaren } 2008 \text { and Southon and } \\
\text { Fedje } 2003\end{array}$ \\
\hline Haida Gwaii & Cybulski 2016 & 265 & \pm 80 & Marine Correction Database ${ }^{f}$ \\
\hline $\begin{array}{l}\text { Dundas } \\
\text { Islands }\end{array}$ & Shugar et al. 2014 & 383 & \pm 172 & $\begin{array}{l}\text { Marine Correction Database for up to } 10 \text { nearest } \\
\text { known values (including Haida Gwaii and } \\
\text { southeast Alaska) }\end{array}$ \\
\hline PRH & Eldridge et al. 2014 & 250 to $400^{\mathrm{g}}$ & - & $\begin{array}{l}\text { Eight shell-wood pairs from stratigraphically intact } \\
\text { archaeological deposits }\end{array}$ \\
\hline PRH & Edinborough et al. 2016 & 273 & \pm 38 & $\begin{array}{l}24 \text { shell-wood pairs (eight each from three separate } \\
\text { contexts) from GbTo-34 }\end{array}$ \\
\hline
\end{tabular}

${ }^{\mathrm{a}} \Delta \mathrm{R}$ values are presented as reported unless the marine reservoir effect was enumerated only as a combined $\mathrm{R}(\mathrm{t})=R+\Delta \mathrm{R}$ value, in which case $\Delta \mathrm{R}$ was derived by subtracting 405 from $\mathrm{R}(\mathrm{t})$, following Reimer et al. 2013.

${ }^{\mathrm{b}}$ Southon and Fedje $(2003: 102)$ estimate $\mathrm{R}(\mathrm{t})=600$ up to $500 \mathrm{BP}$ and 700 thereafter.

${ }^{\mathrm{c}}$ These samples were compiled from museum collections of pre-atomic bomb marine shells in Canada and the United States and reported as data points rather than compiled as $\Delta \mathrm{R}$ values.

${ }^{\mathrm{d}}$ Barron and colleagues (2009:178) propose a marine reservoir effect value of 732 years, though the difference in ages in their Table 1 is 785 years.

'McLaren (2008) presents three independent marine-terrestrial pairs listed here but relies on Southon and Fedje's (2003) estimate of $\mathrm{R}(\mathrm{t})=600$ as accurate.

${ }_{\text {f }}^{\text {See http://calib.org/marine/. }}$

${ }^{\mathrm{g}}$ Eldridge and colleagues (2014:66-67) plot an intercept curve from a trimmed set of eight data points from a sample of nine ( $\Delta \mathrm{R}$ range $=-41$ to -821 , with SD range of \pm 40 to 75 ) with one outlier excluded. No calculation for error for the $\Delta \mathrm{R}$ is presented.

$\Delta \mathrm{R}$; thus their result illustrates both the second method and its limitations.

In contrast, calculating the modeled marine age requires the calibration of the terrestrial age, projecting this range onto the marine calibration curve, and computing the equivalent (modeled) marine age. The process for the intercept method for the Southon and Fedje (2003) example includes the following steps: First, the $1 \sigma$ calibrated age range for the charcoal sample (CAMS-49625, $1560 \pm 40$ ) is $1400-1525$, derived from the IntCal13 curve. This converts the conventional 
Table 2. ${ }^{14} \mathrm{C}$ Marine-Terrestrial Pairings from the Prince Rupert Harbour Study Area.

\begin{tabular}{|c|c|c|c|c|c|c|c|c|c|c|c|c|c|c|}
\hline $\begin{array}{l}\text { Site (Borden } \\
\text { Number) }\end{array}$ & $\begin{array}{c}\text { Terrestrial } \\
\text { Sample Lab } \\
\text { Number }\end{array}$ & Material $^{\mathrm{a}}$ & $\begin{array}{l}\text { Terrestrial } \\
{ }^{14} \mathrm{C} \mathrm{Age}^{\mathrm{b}}\end{array}$ & $\begin{array}{c}\text { Terrestrial } \\
{ }^{14} \mathrm{C} 1 \sigma \\
\text { Error }\end{array}$ & $\begin{array}{l}\text { Marine Sample } \\
\text { Lab Number }\end{array}$ & $\begin{array}{c}\text { Marine } \\
\text { Sample } \\
\text { Material }^{\mathrm{c}}\end{array}$ & $\begin{array}{c}\text { Marine } \\
\text { Sample } \\
{ }^{14} \mathrm{C} \mathrm{Age}^{\mathrm{b}}\end{array}$ & $\begin{array}{l}\text { Marine } \\
{ }^{14} \mathrm{C} 1 \sigma \\
\text { Error }\end{array}$ & $\begin{array}{l}\Delta^{13} \mathrm{C} \\
\text { Value }^{\mathrm{d}}\end{array}$ & $\begin{array}{c}\text { Calibrated } \\
\text { Terrestrial Age }\end{array}$ & $\begin{array}{l}\text { Modeled } \\
\text { Marine Age }\end{array}$ & $\Delta \mathrm{R}^{\mathrm{f}}$ & $\begin{array}{l}\Delta \mathrm{R} 1 \sigma \\
\text { Error }^{\mathrm{f}}\end{array}$ & Source \\
\hline Benke Lagoon & D-AMS 007893 & $\mathrm{~g}$ & 8962 & 32 & D-AMS 007877 & My & 9908 & 33 & -1.2 & $10,224-10,121$ & $9406-9250$ & 576 & 52 & Letham et al. 2018 \\
\hline Benke Lagoon & D-AMS 007894 & $\mathrm{~g}$ & 9359 & 28 & D-AMS 007878 & $\mathrm{Cl}$ & 10,154 & 34 & -7.4 & $10,670-10,506$ & $9768-9615$ & 466 & 54 & Letham et al. 2018 \\
\hline Tea Bay Creek & D-AMS 004469 & $\mathrm{~g}$ & 8472 & 35 & D-AMS 004468 & $\mathrm{Sa}$ & 9526 & 34 & -2.8 & $9533-9447$ & $8910-8769$ & 681 & 49 & Letham et al. 2018 \\
\hline Tea Bay Creek & D-AMS 005846 & $\mathrm{~g}$ & 9559 & 39 & D-AMS 005845 & $\mathrm{c}$ & 9508 & 43 & -1.2 & $11,090-10,730$ & $10,006-9829$ & -445 & 89 & Letham et al. 2018 \\
\hline Tea Bay Creek & D-AMS 005850 & $\mathrm{~g}$ & 9989 & 41 & D-AMS 005851 & ba & 10,256 & 31 & 2.2 & $11,695-11,268$ & $11,479-10,102$ & -137 & 53 & Letham et al. 2018 \\
\hline GbTo-24 & CAMS-49623 & $\mathrm{c}$ & 2040 & 50 & CAMS-49624 & $P r$ & 2780 & 50 & - & $2128-1887$ & $2479-2284$ & 400 & 74 & $\begin{array}{l}\text { Southon and Fedje } \\
\quad 2003\end{array}$ \\
\hline GbTo-24 & CAMS-49625 & $\mathrm{c}$ & 1560 & 40 & CAMS-49626 & $\mathrm{Sa}$ & 2370 & 50 & - & $1541-1367$ & $1988-1834$ & 453 & 65 & $\begin{array}{l}\text { Southon and Fedje } \\
\quad 2003\end{array}$ \\
\hline GbTo-34 & SUERC-44455 & $\mathrm{c}$ & 3359 & 29 & SUERC-44454 & My & 3738 & 29 & -1.5 & $3690-3496$ & $3766-3606$ & 41 & 42 & $\begin{array}{l}\text { Edinborough et al. } \\
\quad 2016\end{array}$ \\
\hline GbTo-34 & SUERC-44455 & $\mathrm{c}$ & 3359 & 29 & SUERC-44456 & My & 4242 & 29 & -0.4 & $3690-3496$ & $3766-3606$ & 545 & 42 & $\begin{array}{l}\text { Edinborough et al. } \\
\quad 2016\end{array}$ \\
\hline GbTo-34 & SUERC-44455 & $\mathrm{c}$ & 3359 & 29 & SUERC-44458 & My & 4043 & 29 & -0.03 & $3690-3496$ & $3766-3606$ & 346 & 42 & $\begin{array}{l}\text { Edinborough et al. } \\
\quad 2016\end{array}$ \\
\hline GbTo-34 & SUERC-44455 & $\mathrm{c}$ & 3359 & 29 & SUERC-44460 & My & 3868 & 27 & -0.7 & $3690-3496$ & $3766-3606$ & 170 & 40 & $\begin{array}{l}\text { Edinborough et al. } \\
\quad 2016\end{array}$ \\
\hline GbTo-34 & SUERC-44457 & $\mathrm{c}$ & 3340 & 29 & SUERC-44456 & My & 4242 & 29 & -0.4 & $3678-3480$ & $3750-3597$ & 564 & 46 & $\begin{array}{l}\text { Edinborough et al. } \\
\quad 2016\end{array}$ \\
\hline GbTo-34 & SUERC-44457 & $\mathrm{c}$ & 3340 & 29 & SUERC-44454 & My & 3738 & 29 & -1.5 & $3678-3480$ & $3750-3597$ & 60 & 46 & $\begin{array}{l}\text { Edinborough et al. } \\
\quad 2016\end{array}$ \\
\hline GbTo-34 & SUERC-44457 & $\mathrm{c}$ & 3340 & 29 & SUERC-44458 & My & 4043 & 29 & -0.03 & $3678-3480$ & $3750-3597$ & 366 & 46 & $\begin{array}{l}\text { Edinborough et al. } \\
\quad 2016\end{array}$ \\
\hline GbTo-34 & SUERC-44457 & $\mathrm{c}$ & 3340 & 29 & SUERC-44460 & My & 3868 & 27 & -0.7 & $3678-3480$ & $3750-3597$ & 190 & 44 & $\begin{array}{l}\text { Edinborough et al. } \\
\quad 2016\end{array}$ \\
\hline GbTo-34 & SUERC-44459 & $\mathrm{c}$ & 3947 & 27 & SUERC-44458 & My & 4043 & 29 & -0.03 & $4515-4294$ & $4379-4205$ & -262 & 48 & $\begin{array}{l}\text { Edinborough et al. } \\
\quad 2016\end{array}$ \\
\hline GbTo-34 & SUERC-44459 & $\mathrm{c}$ & 3947 & 27 & SUERC-44454 & My & 3738 & 29 & -1.5 & $4515-4294$ & $4379-4205$ & -566 & 48 & $\begin{array}{l}\text { Edinborough et al. } \\
\quad 2016\end{array}$ \\
\hline GbTo-34 & SUERC-44459 & $\mathrm{c}$ & 3947 & 27 & SUERC-44456 & My & 4242 & 29 & -0.4 & $4515-4294$ & $4379-4205$ & -62 & 48 & $\begin{array}{l}\text { Edinborough et al. } \\
\quad 2016\end{array}$ \\
\hline GbTo-34 & SUERC-44459 & $\mathrm{c}$ & 3947 & 27 & SUERC-44460 & My & 3868 & 27 & -0.7 & $4515-4294$ & $4379-4205$ & -437 & 47 & $\begin{array}{l}\text { Edinborough et al. } \\
\quad 2016\end{array}$ \\
\hline GbTo-34 & SUERC-44464 & $\mathrm{c}$ & 3106 & 29 & SUERC-44460 & My & 3868 & 27 & -0.7 & $3383-3238$ & $3508-3380$ & 412 & 46 & $\begin{array}{l}\text { Edinborough et al. } \\
\quad 2016\end{array}$ \\
\hline GbTo-34 & SUERC-44464 & $\mathrm{c}$ & 3106 & 29 & SUERC-44454 & My & 3738 & 29 & -1.5 & $3383-3238$ & $3508-3380$ & 282 & 46 & $\begin{array}{l}\text { Edinborough et al. } \\
\quad 2016\end{array}$ \\
\hline GbTo-34 & SUERC-44464 & $\mathrm{c}$ & 3106 & 29 & SUERC-44456 & My & 4242 & 29 & -0.4 & $3383-3238$ & $3508-3380$ & 786 & 46 & \\
\hline
\end{tabular}




\begin{tabular}{|c|c|c|c|c|c|c|c|c|c|c|c|c|c|c|}
\hline $\begin{array}{l}\text { Site (Borden } \\
\text { Number) }\end{array}$ & $\begin{array}{c}\text { Terrestrial } \\
\text { Sample Lab } \\
\text { Number }\end{array}$ & Material $^{\mathrm{a}}$ & $\begin{array}{l}\text { Terrestrial } \\
{ }^{14} \mathrm{C} \mathrm{Age}^{\mathrm{b}}\end{array}$ & $\begin{array}{c}\text { Terrestrial } \\
{ }^{14} \mathrm{C} 1 \sigma \\
\text { Error }\end{array}$ & $\begin{array}{c}\text { Marine Sample } \\
\text { Lab Number }\end{array}$ & $\begin{array}{c}\text { Marine } \\
\text { Sample } \\
\text { Material }^{\mathrm{c}}\end{array}$ & $\begin{array}{c}\text { Marine } \\
\text { Sample } \\
{ }^{14} \mathrm{C} \mathrm{Age}^{\mathrm{b}}\end{array}$ & $\begin{array}{l}\text { Marine } \\
{ }^{14} \mathrm{C} 1 \sigma \\
\text { Error }\end{array}$ & $\begin{array}{l}\Delta^{13} \mathrm{C} \\
\text { Value }^{\mathrm{d}}\end{array}$ & $\begin{array}{c}\text { Calibrated } \\
\text { Terrestrial Age }\end{array}$ & $\begin{array}{c}\text { Modeled } \\
\text { Marine Age }\end{array}$ & $\Delta \mathrm{R}^{\mathrm{f}}$ & $\begin{array}{l}\Delta \mathrm{R} 1 \sigma \\
\text { Error }^{\mathrm{f}}\end{array}$ & Source \\
\hline & & & & & & & & & & & & & & $\begin{array}{l}\text { Edinborough et al. } \\
2016\end{array}$ \\
\hline GbTo-34 & SUERC-44464 & $\mathrm{c}$ & 3106 & 29 & SUERC-44458 & My & 4043 & 29 & -0.03 & $3383-3238$ & $3508-3380$ & 588 & 46 & $\begin{array}{l}\text { Edinborough et al. } \\
\quad 2016\end{array}$ \\
\hline GbTo-34 & SUERC-44466 & $\mathrm{c}$ & 4218 & 29 & SUERC-44465 & My & 4852 & 27 & -0.5 & $4853-4645$ & $4660-4470$ & 238 & 40 & $\begin{array}{l}\text { Edinborough et al. } \\
\quad 2016\end{array}$ \\
\hline GbTo-34 & SUERC-44466 & $\mathrm{c}$ & 4218 & 29 & SUERC-44467 & My & 4898 & 27 & -0.3 & $4853-4645$ & $4660-4470$ & 284 & 40 & $\begin{array}{l}\text { Edinborough et al. } \\
\quad 2016\end{array}$ \\
\hline GbTo-34 & SUERC-44466 & $\mathrm{c}$ & 4218 & 29 & SUERC-44469 & My & 4886 & 29 & -0.3 & $4853-4645$ & $4660-4470$ & 272 & 42 & $\begin{array}{l}\text { Edinborough et al. } \\
\quad 2016\end{array}$ \\
\hline GbTo-34 & SUERC-44466 & $\mathrm{c}$ & 4218 & 29 & SUERC-44474 & My & 4854 & 29 & -1.5 & $4853-4645$ & $4660-4470$ & 240 & 42 & $\begin{array}{l}\text { Edinborough et al. } \\
\quad 2016\end{array}$ \\
\hline GbTo-34 & SUERC-44468 & $\mathrm{c}$ & 4182 & 27 & SUERC-44467 & My & 4898 & 27 & -0.3 & $4836-4620$ & $4630-4454$ & 343 & 74 & $\begin{array}{l}\text { Edinborough et al. } \\
\quad 2016\end{array}$ \\
\hline GbTo-34 & SUERC-44468 & $\mathrm{c}$ & 4182 & 27 & SUERC-44465 & My & 4852 & 27 & -0.5 & $4836-4620$ & $4630-4454$ & 297 & 74 & $\begin{array}{l}\text { Edinborough et al. } \\
\quad 2016\end{array}$ \\
\hline GbTo-34 & SUERC-44468 & $\mathrm{c}$ & 4182 & 27 & SUERC-44469 & My & 4886 & 29 & -0.4 & $4836-4620$ & $4630-4454$ & 344 & 93 & $\begin{array}{l}\text { Edinborough et al. } \\
\quad 2016\end{array}$ \\
\hline GbTo-34 & SUERC-44468 & $\mathrm{c}$ & 4182 & 27 & SUERC-44474 & My & 4854 & 29 & -0.1 & $4836-4620$ & $4630-4454$ & 312 & 93 & $\begin{array}{l}\text { Edinborough et al. } \\
\quad 2016\end{array}$ \\
\hline GbTo-34 & SUERC-44470 & $\mathrm{c}$ & 4176 & 27 & SUERC-44469 & My & 4886 & 29 & -0.4 & $4833-4616$ & $4625-4452$ & 348 & 91 & $\begin{array}{l}\text { Edinborough et al. } \\
\quad 2016\end{array}$ \\
\hline GbTo-34 & SUERC-44470 & $\mathrm{c}$ & 4176 & 27 & SUERC-44465 & My & 4852 & 27 & -0.5 & $4833-4616$ & $4625-4452$ & 314 & 91 & $\begin{array}{l}\text { Edinborough et al. } \\
\quad 2016\end{array}$ \\
\hline GbTo-34 & SUERC-44470 & $\mathrm{c}$ & 4176 & 27 & SUERC-44467 & My & 4898 & 27 & -0.3 & $4833-4616$ & $4625-4452$ & 360 & 91 & $\begin{array}{l}\text { Edinborough et al. } \\
\quad 2016\end{array}$ \\
\hline GbTo-34 & SUERC-44470 & $\mathrm{c}$ & 4176 & 27 & SUERC-44474 & My & 4854 & 29 & -0.1 & $4833-4616$ & $4625-4452$ & 316 & 91 & $\begin{array}{l}\text { Edinborough et al. } \\
\quad 2016\end{array}$ \\
\hline GbTo-34 & SUERC-44475 & $\mathrm{c}$ & 4216 & 27 & SUERC-44474 & My & 4854 & 29 & -0.1 & $4851-4646$ & $4656-4471$ & 291 & 97 & $\begin{array}{l}\text { Edinborough et al. } \\
\quad 2016\end{array}$ \\
\hline GbTo-34 & SUERC-44475 & $\mathrm{c}$ & 4216 & 27 & SUERC-44469 & My & 4886 & 29 & -0.4 & $4851-4646$ & $4656-4471$ & 323 & 97 & $\begin{array}{l}\text { Edinborough et al. } \\
\quad 2016\end{array}$ \\
\hline GbTo-34 & SUERC-44475 & $\mathrm{c}$ & 4216 & 27 & SUERC-44467 & My & 4898 & 27 & -0.3 & $4851-4646$ & $4656-4471$ & 335 & 96 & $\begin{array}{l}\text { Edinborough et al. } \\
\quad 2016\end{array}$ \\
\hline GbTo-34 & SUERC-44475 & $\mathrm{c}$ & 4216 & 27 & SUERC-44465 & My & 4852 & 27 & -0.5 & $4851-4646$ & $4656-4471$ & 289 & 96 & $\begin{array}{l}\text { Edinborough et al. } \\
\quad 2016\end{array}$ \\
\hline GbTo-34 & SUERC-44477 & $\mathrm{c}$ & 1720 & 27 & SUERC-44476 & My & 2239 & 29 & -0.6 & $1701-1561$ & $2120-2012$ & 173 & 61 & \\
\hline
\end{tabular}




\begin{tabular}{|c|c|c|c|c|c|c|c|c|c|c|c|c|c|c|}
\hline GbTo-34 & SUERC-44477 & c & 1720 & 27 & SUERC-44478 & My & 2352 & 29 & -0.9 & $1701-1561$ & $2120-2012$ & 286 & 61 & $\begin{array}{l}2016 \\
\text { Edinborough et al. } \\
2016\end{array}$ \\
\hline GbTo-34 & SUERC-44477 & $\mathrm{c}$ & 1720 & 27 & SUERC-44480 & My & 2409 & 27 & -0.2 & $1701-1561$ & $2120-2012$ & 343 & 60 & $\begin{array}{l}\text { Edinborough et al. } \\
2016\end{array}$ \\
\hline GbTo-34 & SUERC-44477 & c & 1720 & 27 & SUERC-44485 & My & 2274 & 29 & -0.2 & $1701-1561$ & $2120-2012$ & 208 & 61 & $\begin{array}{l}\text { Edinborough et al. } \\
2016\end{array}$ \\
\hline GbTo-34 & SUERC-44479 & $\mathrm{c}$ & 1890 & 27 & SUERC-44478 & My & 2352 & 29 & -0.9 & $1892-1737$ & $2289-2153$ & 131 & 74 & $\begin{array}{l}\text { Edinborough et al. } \\
2016\end{array}$ \\
\hline GbTo-34 & SUERC-44479 & $\mathrm{c}$ & 1890 & 27 & SUERC-44476 & My & 2239 & 29 & -0.6 & $1892-1737$ & $2289-2153$ & 18 & 74 & $\begin{array}{l}\text { Edinborough et al. } \\
2016\end{array}$ \\
\hline GbTo-34 & SUERC-44479 & $\mathrm{c}$ & 1890 & 27 & SUERC-44480 & My & 2409 & 27 & -0.2 & $1892-1737$ & $2289-2153$ & 188 & 73 & $\begin{array}{l}\text { Edinborough et al. } \\
2016\end{array}$ \\
\hline GbTo-34 & SUERC-44479 & $\mathrm{c}$ & 1890 & 27 & SUERC-44485 & My & 2274 & 29 & -0.2 & $1892-1737$ & $2289-2153$ & 53 & 74 & $\begin{array}{l}\text { Edinborough et al. } \\
2016\end{array}$ \\
\hline GbTo-34 & SUERC-44484 & $\mathrm{c}$ & 1619 & 24 & SUERC-44480 & My & 2409 & 27 & -0.2 & $1563-1415$ & 2014-1889 & 458 & 68 & $\begin{array}{l}\text { Edinborough et al. } \\
2016\end{array}$ \\
\hline GbTo-34 & SUERC-44484 & $\mathrm{c}$ & 1619 & 24 & SUERC-44476 & My & 2239 & 29 & -0.6 & $1563-1415$ & 2014-1889 & 288 & 69 & $\begin{array}{l}\text { Edinborough et al. } \\
2016\end{array}$ \\
\hline GbTo-34 & SUERC-44484 & $\mathrm{c}$ & 1619 & 24 & SUERC-44478 & My & 2352 & 29 & -0.9 & $1563-1415$ & 2014-1889 & 401 & 69 & $\begin{array}{l}\text { Edinborough et al. } \\
2016\end{array}$ \\
\hline GbTo-34 & SUERC-44484 & $\mathrm{c}$ & 1619 & 24 & SUERC-44485 & My & 2274 & 29 & -0.2 & $1563-1415$ & 2014-1889 & 323 & 69 & $\begin{array}{l}\text { Edinborough et al. } \\
2016\end{array}$ \\
\hline GbTo-34 & SUERC-44486 & c & 1685 & 29 & SUERC-44485 & My & 2274 & 29 & -0.2 & $1693-1532$ & 2111-1977 & 230 & 73 & $\begin{array}{l}\text { Edinborough et al. } \\
\quad 2016\end{array}$ \\
\hline GbTo-34 & SUERC-44486 & $\mathrm{c}$ & 1685 & 29 & SUERC-44476 & My & 2239 & 29 & -0.6 & $1693-1532$ & $2111-1977$ & 195 & 73 & $\begin{array}{l}\text { Edinborough et al. } \\
2016\end{array}$ \\
\hline GbTo-34 & SUERC-44486 & $\mathrm{c}$ & 1685 & 29 & SUERC-44478 & My & 2352 & 29 & -0.9 & $1693-1532$ & $2111-1977$ & 308 & 73 & $\begin{array}{l}\text { Edinborough et al. } \\
2016\end{array}$ \\
\hline GbTo-34 & SUERC-44486 & c & 1685 & 29 & SUERC-44480 & My & 2409 & 27 & -0.2 & $1693-1532$ & 2111-1977 & 365 & 72 & $\begin{array}{l}\text { Edinborough et al. } \\
2016\end{array}$ \\
\hline GbTo-54 & D-AMS 005136 & $\mathrm{c}$ & 1940 & 34 & D-AMS 005137 & $\mathrm{~s}$ & 2421 & 25 & -5.2 & $1984-1821$ & $2365-2221$ & 128 & 76 & $\begin{array}{l}\text { Eldridge et al. } \\
2014\end{array}$ \\
\hline GbTo-54 & D-AMS 005138 & $\mathrm{c}$ & 1270 & 25 & D-AMS 005139 & $\mathrm{~s}$ & 1894 & 29 & 0.6 & $1281-1176$ & $1731-1612$ & 223 & 66 & $\begin{array}{l}\text { Eldridge et al. } \\
2014\end{array}$ \\
\hline GbTo-54 & D-AMS 005140 & c & 1770 & 29 & D-AMS 005141 & $\mathrm{~s}$ & 2920 & 28 & -0.4 & $1812-1606$ & $2208-2046$ & 793 & 86 & $\begin{array}{l}\text { Eldridge et al. } \\
2014\end{array}$ \\
\hline GbTo-54 & D-AMS 005142 & c & 75 & 28 & D-AMS 005143 & $\mathrm{~s}$ & 875 & 27 & -0.4 & $260-27$ & $598-450$ & 351 & 79 & $\begin{array}{l}\text { Eldridge et al. } \\
2014\end{array}$ \\
\hline GbTo-54 & D-AMS 005145 & $\mathrm{c}$ & 1473 & 33 & D-AMS 005146 & $\mathrm{~s}$ & 2058 & 27 & -0.6 & $1412-1301$ & $1886-1768$ & 231 & 65 & $\begin{array}{l}\text { Eldridge et al. } \\
2014\end{array}$ \\
\hline GbTo-54 & D-AMS 005147 & $\mathrm{c}$ & 2500 & 33 & D-AMS 005148 & $\mathrm{~s}$ & 2930 & 27 & -0.1 & $2738-2466$ & 2969-2757 & 67 & 109 & $\begin{array}{l}\text { Eldridge et al. } \\
2014\end{array}$ \\
\hline
\end{tabular}




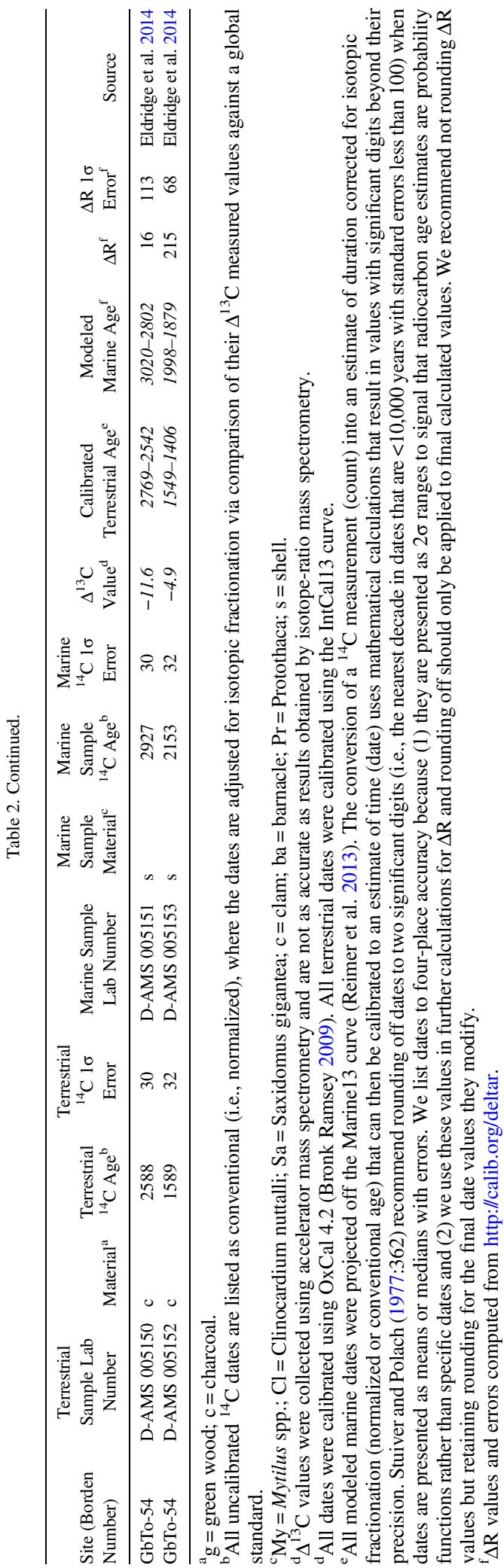

age (in radiocarbon years BP) into a calibrated age range. Second, each of the bracketing ages in this calibrated age range (1400 and 1525 at $1 \sigma$ ) is then projected onto the Marine13 curve to derive an equivalent marine age range (1870 and 1968 years BP, respectively). This converts the calibrated age (years BP) back into a conventional age (in radiocarbon years BP), but interpolated from the marine curve. Third, the mean of the modeled marine age is 1915 years. Fourth, the difference between the conventional marine age (CAMS-49626, 2370 \pm 50 ) and the modeled marine age mean (1915) is 455 years. This is the modeled value of $\Delta R$.

A single value of $\Delta R$ is incomplete without an estimate of its uncertainty. As shown below, the error range in $\Delta \mathrm{R}$ is computed as the square root of the sum of the squares of the conventional marine and modeled marine ages. In this example, the uncertainty calculation follows these steps: First, the $1 \sigma$ error range of the conventional marine age is 50 . Second, the modeled marine error is recorded as half the projected range from the terrestrial age interpolated from the marine curve: $1870-1968=98$, and then $98 / 2=49$. Third, the conventional and modeled errors are combined as the square root of the sum of their squares. Fourth, the sum of the squares of the ages $=50^{2}+49^{2}=4901$. Fifth, the square root of the sum of the squares $=70$ (i.e., uncertainty is \pm 70 ).

Using the deltar calculation tool, this same pair of samples returns a similar result for $\Delta \mathrm{R}$ of $453 \pm 65$. Therefore, the method of computation influences both the value and the error of the $\Delta \mathrm{R}$ value. In this case four different results are derived for methods $1-4$, respectively, of $410-670 \pm 20-70,405 \pm 60,455 \pm 70$, and 435 \pm 65 years. The deltar tool is the easiest and more accurate assessment for computing a $\Delta \mathrm{R}$ value from single pairs of matched marine and terrestrial ages, which we use for our calculations in the PRH example below (see Table 2).

The computation of a difference between a paired marine and terrestrial sample is often insufficient by itself to accurately estimate $\Delta \mathrm{R}$ in archaeological contexts for two reasons. First, a comparison of the measurement, or counting, errors on the terrestrial and marine ages in single-pair samples is routinely, but 
incorrectly, used to generate estimates of uncertainty. Second, in archaeological contexts there is no way to identify chronologically mismatched samples, which can result in considerable inaccuracy. However, both issues can be mitigated with the use of multiple marine and terrestrial sample pairs from the same context.

\section{Addressing Accuracy and Precision of $\Delta R$ with Multiple Marine-Terrestrial Pairs}

Cook and colleagues (2015:165) identify concerns with the single-pair sample approach in which $\Delta \mathrm{R}$ is calculated from the difference between a conventional marine age from a marine-derived sample and a modeled marine age calculated from a paired terrestrial sample. They note that such calculations routinely derive from a comparison of mean ages rather than more realistic estimates of uncertainty. They demonstrate that randomly generated values within the standard deviations of the conventional ages (i.e., values that are statistically indistinguishable from the mean values) increase the range of calculated uncertainty of $\Delta \mathrm{R}$ from a single pair of samples. As illustrated by Cook and colleagues, paired ages from marine $(6500 \pm 80)$ and terrestrial $(6000 \pm 70$, producing a modeled marine age of $6420 \pm 70$ ) samples result in an apparent $\Delta \mathrm{R}$ of 80 years. However, $\Delta \mathrm{R}$ values derived from four randomly generated ages within the standard deviations of the marine and terrestrial ages (i.e., 16 possible pairs) produced a range in $\Delta \mathrm{R}$ of 514 years $(-253$ to +261$)$.

Thus, the uncertainty of $\Delta \mathrm{R}$ from single-pair samples is generally much greater than archaeologists estimate by relying on measurement errors in single-pair assessments. Originally, Russell and colleagues (2010:1171) proposed that the error on the $\Delta \mathrm{R}$ value could be calculated as the square root of the sum of the squares of the errors on the measured marine and terrestrial ages. Recently, Cook and colleagues (2015:166) have argued that this calculation does not include the uncertainty that would encompass any future individual measurements of $\Delta \mathrm{R}$ made on a single pair of samples from the measured context. Thus, they propose a revised estimate of uncertainty in $\Delta \mathrm{R}$ as the standard error for predicted values: the square root of the sum of the squares of (1) the error on the weighted mean of the $\Delta \mathrm{Rs}$ from all possible sample pairings and (2) the standard deviation of the $\Delta \mathrm{R}$ values. This can be calculated with the following steps: First, using the steps outlined in the previous section, calculate $\Delta \mathrm{R}$ values of interest from multiple paired samples. Second, compute the weighted mean of the $\Delta \mathrm{R}$ values (see Ward and Wilson 1978). Since we are assuming that the $\Delta \mathrm{R}$ estimates derive from a coherent population, for example, Ward and Wilson's (1978:20-21) Case I, we use their equation 1: the weighted (aka pooled) mean $=$ the sum of all the age estimates divided by the squared standard error and then divided by the sum of the inverse of all squared standard errors (see the supplemental data for a Microsoft Excel spreadsheet template for this calculation). Third, compute the standard deviation of the $\Delta \mathrm{R}$ values. Fourth, the standard error for predicted values is the square root of the sum of the squares of the error on the weighted mean and the standard deviation of the $\Delta \mathrm{R}$ values (see the supplemental data for a Microsoft Excel spreadsheet template for this calculation).

The key message for archaeologists is that single-pair assessments of $\Delta \mathrm{R}$ are less certain than the commonly applied archaeological calculations of uncertainty. This creates a paradox in which archaeologists either underestimate actual uncertainty, thereby generating overly precise values that are demonstrably inaccurate, or correctly estimate uncertainty, thereby generating accurate values that have such imprecision as to make marine-sourced samples considerably less useful for building chronologies.

Russell and colleagues (2011), following Ascough and colleagues (2007), propose that multiple-pair samples (i.e., four marine and four terrestrial samples) taken from the same stratigraphic context provide an opportunity to reduce mismatching and generate a more accurate estimate of $\Delta \mathrm{R}$ than single-pair samples. Each set of four marine and terrestrial samples can be evaluated for consistency via a chi-square test. Failure indicates mismatching in the marine or terrestrial samples. If a single outlier date is the source of the inconsistency, then it can be excluded, and the remaining ages may be used if they pass a chi-squared test. A chi-square test permits an assessment of coherence within a 
pool of data, in effect asking the probability that the sample data derive from a homogeneous population. In this context, the population is a suite of radiocarbon ages that would be produced if the terrestrial and marine samples were drawn from organisms that died at the same time. This expectation creates a model in which the terrestrial and marine values would be similar to each other, and thus the difference between them would be consistent. This mathematical pattern can be projected as a statistically expected value; the chi-square test evaluates the difference between the observed data and the expected value via a $T$-statistic (Wilson and Ward 1981:20). Ward and Wilson (1978) and Wilson and Ward (1981) present the mathematics of assessing the coherence of a set of dates as the sum of the squared differences between each age and the weighted mean of the set divided by the square of the $1 \sigma$ error value (see the supplemental data for a Microsoft Excel template): First, calculate the weighted mean for the measured (i.e., uncalibrated) ages of the marine or terrestrial samples using the steps listed above. Second, subtract each measured age from the weighted mean, square this value, and divide it by the square of the measured error for that sample. Third, sum these results. The resulting chi-square value is large if the variation between ages is large and small if it is small. Fourth, the chi-square value can be compared with an expected result (a $T$-value) derived from the chi-square function and the degrees of freedom in the calculation. In our case, with four samples, the degrees of freedom are three, and the acceptance level of the $T$-value is 7.815. A calculated chi-square value above this level is a fail (i.e., the samples have a $>95 \%$ chance of not being from the same population). Fifth, if the failure is due to an obvious outlier date, this can be dropped and the test can be rerun. Edinborough and colleagues (2016) accept a marginal fail from one context with one outlier removed. However, a failure means that the samples are mismatched and cannot be used to compute a $\Delta \mathrm{R}$. Additional samples may need to be collected and new dates may need to be assessed until they pass the chi-square test. There is some subjectivity here in relation to the number of outliers; the context could contain samples of mixed ages that cannot provide a coherent result.

While the chi-square assessment is more expensive than using single pairs, its ability to identify mismatching is invaluable. Mismatched samples are likely a significant source of variation in $\Delta \mathrm{R}$ values from single-pair tests, but mismatching can only be identified through multiple-sample testing. Taking multiple samples from identical contexts also increases the number of $\Delta \mathrm{R}$ results per ${ }^{14} \mathrm{C}$ age measurement. When individual pairs are taken in different contexts, the ratio of $\Delta \mathrm{R}$ estimates per ${ }^{14} \mathrm{C}$ measurement is $1: 2$, while this ratio increases to $2: 1$ with four marine and four terrestrial samples from identical contexts. This makes more efficient use of ${ }^{14} \mathrm{C}$ measurements and consequently of research budgets as well.

As we demonstrate below, increasing the sample size via a simple amplification of the singlepair approach does not guarantee increased precision, likely because of mismatched pairings. The corollary of this logic is that many extant archaeological estimates of $\Delta \mathrm{R}$ underestimate uncertainty and are potentially inaccurate.

\section{The Prince Rupert Harbour Example}

An archaeological view of analyses of MRE conditions and $\Delta \mathrm{R}$ calculations is often parochial and focused on making optimal use of radiocarbon dates from marine-sourced samples in specific contexts. As archaeologists frequently attempt to retrospectively obtain the most accurate and precise age estimate from their marine-derived samples, $\Delta \mathrm{R}$ analyses and calculations are typically conducted long after samples have been collected and dated. Many archaeologists rely on regional estimates of $\Delta \mathrm{R}$ rather than conducting local tests. Although such tests are becoming more common, the cost of increasing the marineterrestrial sample size to refine $\Delta \mathrm{R}$ estimates is often considered prohibitive in the context of other research objectives. Our own research illustrates this. Prior to our work, regional and local estimates of $\Delta R$ existed, based on small numbers of single-pair samples (Table 1). Most presented $\Delta \mathrm{R}$ as a constant, but some (Eldridge et al. 2014; McKechnie and Eldridge 2013; Southon and Fedje 2003) presented evidence of changes 
over time. Our settlement history research (see Ames and Martindale 2014; Letham et al. 2015; Martindale, Letham, et al. 2017; Martindale, Marsden, et al. 2017) relies heavily on the dating of shell samples; thus we developed a $\Delta \mathrm{R}$ test in concert with the Scottish Universities Environmental Research Centre to apply the approach of Russell and colleagues (2011) to the PRH, which produced a statistically robust result spanning the last 5,000 years (Edinborough et al. 2016). Here we consider this result in comparison to both (1) the history of $\Delta \mathrm{R}$ estimates in the PRH region and (2) commonly applied but inaccurate and imprecise calculations illustrated with our data. We then discuss the implications of these options for archaeology.

\section{PRH Archaeology}

As in many coastal areas, the archaeology of the $\mathrm{PRH}$ region (Figure 1) is complex and presents both a range of archaeological components and a legacy of variation in materials dated. The primary material for site formation is marine shell, and the primary diet for humans is marine-sourced, including invertebrates, fish, and sea mammals. Isotopic analyses of human diet (Chisholm et al. 1983; Cybulski 2016; Schwarcz et al. 2014) and quantitative zooarchaeology (Coupland et al. 2010; Stewart et al. 2009) independently identify a diet ranging between $85 \%$ and $100 \%$ marine-derived protein.

Shell-bearing sites are ubiquitous in the PRH area and reflect both the by-product of food consumption and engineering efforts to construct level, well-drained habitation terraces in a highly crenulated coastal landscape dominated by bedrock outcrops, gravel beaches, and estuaries (Letham et al. 2017). While dedicated construction episodes are known, in which massive anthropogenic landforms were built in short periods of time, shell-bearing sites also captured recurring daily behaviors, such that their lower and upper surfaces approximate the initiation and termination of occupation, respectively. Regional settlement patterns can be captured in large samples of dated basal and terminal components of marine shell, the former of which are increasingly becoming accessible via percussion coring (Cannon 2000; Letham et al. 2015;
Martindale et al. 2009; McKechnie 2015; Pluckhahn et al. 2015).

The PRH area (approximately $180 \mathrm{~km}^{2}$ ) has a rich archaeological record comprising 157 currently recorded shell middens, 63 of which have architectural surface features and are classified as Tsimshian villages (Ames and Martindale 2014:145). The PRH has seen archaeological research spanning the last century. The current ${ }^{14} \mathrm{C}$ dataset for the PRH region, including archaeological and geologic contexts, spans more than $10,000{ }^{14} \mathrm{C}$ years and includes 200 charcoal/terrestrial plant remains, 288 marine shells/ bones, and 88 dates obtained from human bone collagen (Cybulski 2016; Martindale, Letham, et al. 2017). Our current research focuses on refining the PRH settlement pattern history for the last 6,000 years, a goal that required initiation and termination dates of a representative sample of village sites, as well as developing midden formation chronologies and accumulation rates for a subset of middens. The focus of this work, conducted in partnership with the Lax Kw'alaams and Metlakatla First Nations, has been the comparison of archaeological and Tsimshian oral records, for which we needed accurate chronologies for academic and legal contexts (Edinborough et al. 2017; Martindale, Letham, et al. 2017; Martindale, Marsden, et al. 2017).

\section{Previous Calculations of $\Delta R$ in the PRH Area}

A range of $\Delta \mathrm{R}$ values has been proposed for the PRH area and environs (195 to 670 years), most with a relatively narrow margin of error $( \pm 25-50$ years), based on simply combining the measurement errors of the samples (Table 1). Several authors plot curves from sets of $\Delta \mathrm{R}$ values over time (Eldridge et al. 2014; McKechnie and Eldridge 2013; Southon and Fedje 2003) as a means of estimating a regional $\Delta \mathrm{R}$ trend. For example, Southon and Fedje (2003) propose a regional $R(t)$ of $600 \pm 100$ years for the Haida Gwaii area for post-500 years BP, from a cubic polynomial trend line of least squares of values ranging from 500 to $10,000+$ years ago. A few authors have followed Deo and colleagues (2004) in calculating $\Delta \mathrm{R}$ for different temporal bins (Lepofsky et al. 2015; Martindale et al. 2009), and a few have pooled sets of marineterrestrial pairs within regions, in an attempt to 
increase precision. For example, Carlson (2012) and Carlson and Baichtal (2015:125) propose a $\Delta \mathrm{R}$ of $545 \pm 60$ years, based on four marineterrestrial pairs from different sites in southeast Alaska. Barron and colleagues (2009) propose a $\Delta \mathrm{R}$ of 327 years based on three marineterrestrial pairs from deepwater marine sediment cores farther north in the Gulf of Alaska. Shugar and colleagues (2014) present a regional value for the nearby Dundas Islands of $373 \pm 172$ years, based on a weighted mean of the 10 nearest marine-terrestrial pairs (including some PRH data) calculated from the Marine Reservoir Correction Database, http://calib.org/marine/ (Reimer et al. 2004).

It is reasonable to expect $\mathrm{MRE}$ and $\Delta \mathrm{R}$ to vary considerably across this region and through the time period (Table 1), primarily from the midto late Holocene. Hutchinson (2014) argues that $\Delta \mathrm{R}$ values on the Pacific coast of North America are sensitive to oceanographic factors that vary seasonally and spatially, including wind, currents (including upwelling and downwelling), and coastal configurations (see also Southon et al. 1990). Following Thomson (1981), he identifies two broad patterns along the West Coast: a downwelling zone along the Pacific coast of southeast Alaska from Dixon Inlet to the far end of the Aleutian archipelago and an upwelling zone from the central British Columbia coast to southern Baja, California. In these areas, variations in $\Delta \mathrm{R}$ values are significantly induced by wind and water movements and are affected by El Niño-Southern Oscillation events. Hutchinson predicts that $\Delta \mathrm{R}$ values should be stable in a "transitional zone" between the major downwelling and upwelling zones that includes the Haida Gwaii archipelago and the eastward British Columbia mainland, including PRH (see also Chang et al. 2008).

The variation in $\Delta \mathrm{R}$ values (Table 1) also likely captures patterns resulting from small sample sizes and mismatched pairs. In the absence of multiple-pair testing and chi-square assessments, there is no easy way to determine whether variation is a result of mismatching or MRE. Thus, Table 1 illustrates a problem common to archaeologists working with marine-sourced ${ }^{14} \mathrm{C}$ dates: proposed $\Delta \mathrm{R}$ values in different studies show a high degree of variation but are presented individually as reasonably accurate proxies for MRE.
The choice for archaeologists dating marinesourced carbon is between a coarse regional estimate or a significant financial investment in radiocarbon dates to generate a more correct local value. This creates a significant impediment to using marine-sourced dates in archaeological chronologies.

\section{Calculating $\triangle R$ in PRH}

Unfortunately, simply increasing the sample size of marine-terrestrial pairs in a local area does not guarantee increased accuracy or precision. The PRH area has a large number $(n=63)$ of marineterrestrial ${ }^{14} \mathrm{C}$ pairs (Table 2 ), an order of magnitude larger than for most $\Delta \mathrm{R}$ estimates elsewhere in coastal British Columbia. Thus, it is a good candidate both for estimating $\Delta \mathrm{R}$ and for comparing differing archaeological calculations. As we discuss below, not all of these are suitable for an accurate assessment of MRE. However, archaeologists routinely address uncertainty by increasing the number of single-sample pairs, the limitations of which are easily demonstrated. A plot of the means of all modeled marineterrestrial pairs in the PRH (Figure 2) shows considerable variation over time and between different datasets from specific archaeological sites. These values have a very wide range, -566 to +812 , illustrating the point that, in archaeological contexts, $\Delta \mathrm{R}$ does not necessarily trend toward modality as single-pair sample size increases, particularly across long time spans. As noted above, several factors may be at play in this scattered pattern, including mixed-age samples, old wood/shell effects, and variation in laboratory measurement, as well as the possibility noted in Hutchinson and colleagues (2004) that MRE is heterogeneous over time and space (see also Ingram and Southon 1996).

Pooling all of these pairs produces a weighted mean of 243 years. Using Cook and colleagues' (2015) revised calculation of standard error for predicted values generates a $\Delta R$ value of $243 \pm$ 352 , which is too uncertain to be of much use in archaeological chronologies. Data from before 8000 cal BP are both poorly sampled and highly variable. Excluding these values generates a $\Delta \mathrm{R}$ of $237 \pm 331$, which is only a marginal improvement. Note that if we were to follow the common archaeological habit of estimating $\Delta \mathrm{R}$ as a mean 
of both the values and the measured errors, we could return a result of $247 \pm 49$ years for the last 8,000 years, but this value would be an inaccurate estimate of $\Delta \mathrm{R}$ and its precision.

Some subsets of the data show clear trends that have been reported as significant patterns in MRE. For example, Eldridge and colleagues (2014:67) calculated a linear regression for eight marine-terrestrial pairs from Casey Point (GbTo-54) that show a linear trend in $\Delta \mathrm{R}$ that increases from about 0 to 400 years from about 3,000 years ago to the present. There is a single outlier $(\Delta \mathrm{R}=\sim 800$ years at about $2000 \mathrm{BP})$, which when discarded produced a very high $\mathrm{R}^{2}$ value (0.95) for predicting the slope of the remaining values. In the context of other PRH data, this pattern is considerably weakened, though it is possible that $\Delta \mathrm{R}$ may vary less at specific site locations. If MRE varies in different parts of the PRH, then the challenge of estimating MRE is considerable. However, it is likely that mismatching of marine and terrestrial samples is the source of variation in this dataset, something that can be assessed via multiple-pair samples.

\section{Calculating $\Delta R$ from Multiple-Pair Samples}

Following Russell and colleagues (2011), we dated four marine and four terrestrial samples from each of three stratigraphically intact archaeological contexts at the PRH site of Kitandach (GbTo-34; see Edinborough et al. 2016). We selected four samples from paired charcoal and short-lived marine shell fragments (Mytilus spp., likely Mytilus trossulus) from three stratigraphic components (basal, terminal, and a midcomponent transition) collected via percussion coring. Our results would be improved had we identified plant species of the charcoal samples and retained only short-lived ones. Dating of the samples generated 24 dates in total and 16 potential pairs of dates from each context-thus 48 pairs in total (Table 2).

As presented in Edinborough and colleagues (2016), the four terrestrial and four marine samples from each stratigraphic context were evaluated via chi-square tests to assess their coherence. Only one (CT2012-005, representing the basal layer of the site) passed the chi-square test, producing a $\Delta \mathrm{R}$ as a weighted mean of
$267 \pm 45$ at a mean terrestrial age of $4735 \pm 107$ cal BP. Removal of one marine and one terrestrial outlier from the upper layer of the site (CT2012-020) increased conformity in this set to a marginal fail, with a $\Delta \mathrm{R}$ weighted mean of $288 \pm 69$ at a mean terrestrial age of $1637 \pm 76$ cal BP. The third set from the middle of the site (CT2012-001), which had a mean terrestrial age of $3722 \pm 95 \mathrm{cal} \mathrm{BP}$, was too varied to produce a $\Delta \mathrm{R}$ value under the criteria of Russell and colleagues (2011). Edinborough and colleagues (2016) chose the conservative route of discarding context CT2012-001 and computing a weighted mean of the results, with the error as the standard error for predicted values from CT2012-005 and CT2012-020 $(\Delta \mathrm{R}=273 \pm 38)$ as the revised value of $\Delta \mathrm{R}$ for the PRH.

Given the range of proposed values for a $\mathrm{PRH} \Delta \mathrm{R}$ and the mathematical options for its calculation, it is useful to compare results from alternate methods (Table 3). Here we illustrate the difference between a common archaeological approach to estimating $\Delta \mathrm{R}$ using simple means of age differences and measured values against the use of weighted means and the standard error of predicted values (from Cook et al. 2015). Note that the different $\Delta \mathrm{R}$ estimates are in reasonable accord with many of the regional estimates in Table 1, including the means of these proposed values and their measurement errors. The critical difference between these methods is in the way error is calculated, which has major implications for the accuracy of calibrated ages. A simple mean of measured errors from the PRH (Table 3) generates reasonably precise values $( \pm 66$ for the entire sample, \pm 49 for the more coherent pre-8,000 RCYBP sample). However, applying the standard error of predicted values generates a far greater, and we argue more accurate, estimate of uncertainty $( \pm 352$ for the entire sample, \pm 331 for the more coherent pre-8000 BP sample). These values are both far larger than most archaeological estimates (Table 1) and so large as to make dates from marine samples problematic in the construction of archaeological chronologies. The correct error estimate accurately reflects the heterogeneity of the sample pairs, likely a result of mismatched pairing. While we cannot control for vagaries in MRE over time, we can control for mismatching errors. Thus, 
Table 3. Alternate Estimates of $\Delta \mathrm{R}$ in the Prince Rupert Harbour (PRH) Area.

\begin{tabular}{|c|c|c|c|}
\hline Method & $\begin{array}{c}\Delta \mathrm{R} \\
\text { (years) }\end{array}$ & Error Estimate Calculation & $\begin{array}{l}\text { Error } \\
\text { (years) }\end{array}$ \\
\hline Mean of all regional values from Table 1 & 347 & $\begin{array}{l}\text { Mean of proposed measurement } \\
\text { errors }\end{array}$ & \pm 83 \\
\hline Mean of all $63 \mathrm{PRH}$ pairs & 245 & Mean of measurement errors & \pm 66 \\
\hline Weighted mean of all $63 \mathrm{PRH}$ pairs & 243 & $\begin{array}{l}\text { Standard error of predicted values } \\
\quad(\text { Cook et al. 2015) }\end{array}$ & \pm 352 \\
\hline Mean of $58 \mathrm{PRH}$ pairs dating after $8,000 \mathrm{RCYBP}$ & 247 & Mean of measurement error & \pm 49 \\
\hline Weighted mean of $58 \mathrm{PRH}$ pairs dating after $8,000 \mathrm{RCYBP}$ & 237 & $\begin{array}{l}\text { Standard error of predicted values } \\
\quad(\text { Cook et al. 2015) }\end{array}$ & \pm 331 \\
\hline $\begin{array}{l}\text { Weighted mean of } 16 \text { multiple-pair samples from the PRH that } \\
\text { passed chi-square conformity test from two stratigraphic locations } \\
\text { dating to after 5,000 RCYBP (from Edinborough et al. 2016) }\end{array}$ & 273 & $\begin{array}{l}\text { Standard error of predicted values } \\
\quad \text { (Cook et al. 2015) }\end{array}$ & \pm 38 \\
\hline
\end{tabular}

the multiple-sample method using chi-square tests of conformity to ensure matching and exclude mismatching results in a correct error estimate of only \pm 38 . This revised value is in line with other results developed for the region (Table 1) but is more accurate, as it is based on (1) the assessment of coherence from multiple pairs derived from the same stratigraphic context, (2) $\Delta \mathrm{R}$ values computed via marinemodeled ages of calibrated terrestrial dates, (3) a weighted mean of the resulting values for $\Delta R$ to best approximate a constant over time, and (4) the use of Cook and colleagues' (2015) standard error for predicted values. We argue that these methods of both deriving and calculating estimates of $\Delta \mathrm{R}$ should become standard in future archaeological assessments of $\Delta R$.

\section{Conclusions}

The challenge for archaeologists using marinesourced ${ }^{14} \mathrm{C}$ age measurements is finding a balance between accuracy (the proximity of results to reality) and precision (the range of uncertainty in results) in the estimate of marine reservoir effect via $\Delta R$. Achieving this balance is possible with the multiple-pair approach derived from Ascough and colleagues (2007), Russell and colleagues (2011), and Cook and colleagues (2015). This method requires more investment by archaeologists in radiocarbon dating of marineterrestrial ${ }^{14} \mathrm{C}$ pairs. However, it is more efficient and cost-effective than simply increasing marineterrestrial pair sample sizes, which often exacerbates variability between $\Delta R$ estimates, because it cannot control for mismatched samples. Research budgets and overarching chronological objectives of archaeological projects in coastal settings may have to be adjusted at the project planning and grant application stage as a result. However, the benefits include improved chronological resolution and the fact that a large number of existing marine shell ${ }^{14} \mathrm{C}$ measurements can be appropriately recalibrated and incorporated into regional chronologies. The PRH example indicates that the analytical benefits are clear, as the multiplepair method provides a relatively affordable solution that generates a statistically robust result in comparison with single paired samples. Given the variability in our results, we are cautious in extending the temporal range for our calculated $\Delta \mathrm{R}$. We have insufficient data to propose a $\Delta \mathrm{R}$ for before 5,000 years ago. For these time periods (e.g., Letham et al. 2016), we use the value for the last 5,000 years with the caveat that new data from the terminal Pleistocene and early Holocene are likely to modify it. Finally, archaeologists would be well served by engaging with geologic and geochemical scholars as they propose and refine the use of $\Delta \mathrm{R}$ to estimate MRE for archaeological research.

\section{Note}

1. Deo and colleagues (2004) use the global MRE constant ( 400 years) as 0 in their calculations.

Acknowledgments. This research was conducted with the support and partnership of the Lax Kw'alaams and Metlakatla Indian Bands and the nine hereditary tribes they represent; we are most grateful for permission to work on their lands and 
history. Funding for this research was provided by the National Science Foundation (US) grant \#1216847 (principal investigator: K. Ames) and the Social Science and Humanities Research Council of Canada grant \#410-2011-0841 (principal investigator: A. Martindale). Canadian National Rail is thanked for its financial and other assistance to Millennia Research Limited to complete the excavation and analysis of the Casey Point sites. We also thank T. J. Brown, Bryn Letham, and Michael Richards for providing much useful guidance during the planning and editing of this article. We thank Paula Reimer for access to the intercept concatenation tables and a prepublished version of the announcement of deltar. We are grateful to the three anonymous reviewers whose careful assessment of this work improved it considerably. Many thanks to Luis Borrero for the Spanish translation of the abstract.

Data Availability Statement. The radiocarbon data on which this analysis is based are included within this publication as supplemental material and in Table 2.

Supplemental Materials. For supplementary material accompanying this article, visit https://doi.org/10.1017/aaq. 2018.47.

The supplemental materials accompanying this article are data and formulae in an MS Excel spreadsheet containing radiocarbon dates and the calculation of $\Delta \mathrm{R}$ and Standard Error (Sheet 1) and the multiple paired samples of radiocarbon dates and the calculation of weighted means and chi-square values (Sheet 2).

\section{References Cited}

Ames, Kenneth M.

2005 The North Coast Prehistory Project Excavations in Prince Rupert Harbour, British Columbia: The Artifacts. BAR International Series 1342. British Archaeological Reports, Oxford.

Ames, Kenneth M., and Andrew Martindale

2014 Rope Bridges and Cables: A Synthesis of Prince Rupert Harbour Archaeology. Canadian Journal of Archaeology 38(1):140-178.

Archer, David

1992 The Results of the Prince Rupert Harbour Dating Project. British Columbia Archaeology Branch, Victoria, British Columbia, Canada.

2001 Village Patterns and the Emergence of Ranked Society in the Prince Rupert Area. In Perspectives on Northern Northwest Coast Prehistory, Vol. 160, edited by Jerome Cybulski, pp. 203-222. Archaeology Survey of Canada, Canadian Museum of Civilization, Ottawa, Ontario.

Ascough, Philippa, Gordon Cook, Mike Church, Andrew Dugmore, Thomas McGovern, Elaine Dunbar, Adolf Frioriksson, and Hildur Gestsdóttir

2007 Reservoirs and Radiocarbon: ${ }^{14} \mathrm{C}$ Dating Problems in Mývatnssveit, Northern Iceland. Radiocarbon 49 (2):947-961.

Ascough, Philippa, Gordon Cook, Andrew Dugmore, John Barber, Elaine Higney, and E. Marian Scott

2004 Holocene Variations in the Scottish Marine Radiocarbon Reservoir Effect. Radiocarbon 46(2):611-620.

Barron, John, David Bukry, Walter Dean, Jason Addison, and Bruce Finney
2009 Paleoceanography of the Gulf of Alaska during the Past 15,000 Years: Results from Diatoms, Silicoflagellates, and Geochemistry. Marine Micropaleontology 72(3):176-195.

Bronk Ramsey, Christopher

2009 Bayesian Analyses of Radiocarbon Dates. Radiocarbon 51(1):337-360.

Burley, David, Kevan Edinborough, Marshall Weisler, and Jian-xin Zhao

2015 Bayesian Modeling and Chronological Precision for Polynesian Settlement of Tonga. PLOS ONE 10(3): e0120795. DOI:10.1371/journal.pone.0120795, accessed June 17, 2017.

Cannon, Aubrey

2000 Settlement and Sea-Levels on the Central Coast of British Columbia: Evidence from Shell Midden Cores. American Antiquity 65(1):67-77.

Carlson, Risa

2012 A Predictive Model for Early Holocene Archaeological Sites in Southeast Alaska Based on Elevated Palaeobeaches. PhD dissertation, Department of Archaeology, University of Cambridge, Cambridge.

Carlson, Risa, and James Baichtal

2015 A Predictive Model for Locating Early Holocene Archaeological Sites Based on Raised Shell-Bearing Strata in Southeast Alaska, USA. Geoarchaeology 30 (2): $120-138$

Chang, Alice, Thomas Pedersen, and Ingrid Hendy

2008 Late Quaternary Paleoproductivity History on the Vancouver Island Margin, Western Canada: A Multiproxy Geochemical Study. Canadian Journal of Earth Sciences 45(11):1283-1297.

Chisholm, Brian, D. Erle Nelson, and Henry Schwarcz

1983 Marine and Terrestrial Protein in Prehistoric Diets on the British Columbia Coast. Current Anthropology 24 (3):396-398.

Cook, Gordon, Philippa Ascough, Clive Bonsall, W. Derek Hamilton, Nicola Russell, Kerry Sayle, E. Marian Scott, and Jessica Bownes

2015 Best Practice Methodology for ${ }^{14} \mathrm{C}$ Calibration of Marine and Mixed Terrestrial/Marine Samples. Quaternary Geochronology 27:164-171.

Coupland, Gary, Kathlyn Stewart, and Katherine Patton

2010 Do You Never Get Tired of Salmon? Evidence for Extreme Salmon Specialization at Prince Rupert Harbour, British Columbia. Journal of Anthropological Archaeology 29(2):189-207.

Cybulski, Jerome

2016 Updating the Warrior Cache: Timing the Evidence for Warfare at Prince Rupert Harbour. In Violence and Warfare among Hunter-Gatherers, edited by Mark W. Allen and Terry L. Jones, pp. 333-350. Left Coast Press, Walnut Creek, California.

Deo, Jennie N., John O. Stone, and Julie K. Stein

2004 Building Confidence in Shell: Variations in the Marine Radiocarbon Reservoir Correction for the Northwest Coast over the Past 3,000 Years. American Antiquity 69 (4):771-786

Edinborough, Kevan, Andrew Martindale, Gordon T. Cook, Kisha Supernant, and Kenneth M. Ames

2016 A Marine Reservoir Effect $\Delta \mathrm{R}$ Value for Kitandach, in Prince Rupert Harbour, British Columbia, Canada. Radiocarbon 58(4):885-891.

Edinborough, Kevan, Marko Porčić, Andrew Martindale, Thomas Jay Brown, Kisha Supernant, and Kenneth M. Ames 
2017 Radiocarbon Test for Demographic Events in Written and Oral History. Proceedings of the National Academy of Sciences 114(47):12436-12441.

Eldridge, Morley, Alyssa Parker, Christine Mueller, and Susan Crockford

2014 Archaeological Investigations at Ya asqalu'i/Kaien Siding, Prince Rupert Harbour. Report for the British Columbia Archaeology Branch, Victoria, Government of British Columbia, British Columbia, Canada.

Etayo-Cadavid, Miguel F., C. Fred T. Andrus, Kevin B. Jones, Gregory W. L. Hodgins, Daniel H. Sandweiss, Santiago Uceda-Castillo, and Jeffrey Quilter

2013 Marine Radiocarbon Reservoir Age Variation in Donax obesulus Shells from Northern Peru: Late Holocene Evidence for Extended El Niño. Geology 41(5):599-602.

Gómez, Eduardo A., C. Marcela Borel, Marina L. Aguirre, and Daniel E Martínez

2008 Radiocarbon Reservoir Ages and Hardwater Effect for the Northeastern Coastal Waters of Argentina. Radiocarbon 50(1):119-129.

Goodfriend, Glenn A., and Karl W. Flessa

1997 Radiocarbon Reservoir Ages in the Gulf of California: Roles of Upwelling and Flow from the Colorado River. Radiocarbon 39(2):139-148.

Heier-Nielsen, Susanne, Jan Heinemeier, Henrik L. Nielsen, and Niels Rud

1995 Recent Reservoir Ages for Danish Fjords and Marine Waters. Radiocarbon 37(3):875-882.

Hua, Quan, Gregory E. Webb, Jian-xin Zhao, Luke D. Nothdurft, Matthew Lybolt, Gilbert J. Price, and Bradley N. Opdyke

2015 Large Variations in the Holocene Marine Radiocarbon Reservoir Effect Reflect Ocean Circulation and Climatic Changes. Earth and Planetary Science Letters 422:33-44.

Hughen, Konrad A., Mike G. L. Baillie, Edouard Bard, J. Warren Beck, Chanda J. H. Bertrand, Paul G. Blackwell, Caitlin E. Buck, George S. Burr, Kirsten B. Cutler, and Paul E. Damon

2004 Marine04 Marine Radiocarbon Age Calibration, 0-26 cal kyr BP. Radiocarbon 46(3):1059-1086.

Hutchinson, Ian

2014 The Old Shell Game: Late Holocene Variations in the Marine Reservoir Effect in the Northeast Pacific. Paper presented at the 67th Northwest Anthropological Conference, Bellingham, Washington.

Hutchinson, Ian, Thomas S. James, Paula J. Reimer, Brian D. Bornhold, and John J. Clague

2004 Marine and Limnic Radiocarbon Reservoir Corrections for Studies of Late- and Postglacial Environments in Georgia Basin and Puget Lowland, British Columbia, Canada and Washington, USA. Quaternary Research 61(2):193-203.

Ingram, B. Lynn, and John R. Southon

1996 Reservoir Ages in Eastern Pacific Coastal and Estuarine Waters. Radiocarbon 38(3):573-582.

Kennett, Douglas J., B. Lynn Ingram, Jon M. Erlandson, and Phillip Walker

1997 Evidence for Temporal Fluctuations in Marine Radiocarbon Reservoir Ages in the Santa Barbara Channel, Southern California. Journal of Archaeological Science 24(11):1051-1059.

Kintigh, Keith W.

2015 Concluding Address: Ruminations on Mathematics in Archaeology. In Mathematics and Archaeology, edited by J. A. Barcelo and I. Bogdanovic, pp. 487490. CRC Press, Boca Raton, Florida.

Kovanen, Dori J., and Don J. Easterbrook

2002 Paleodeviations of Radiocarbon Marine Reservoir Values for the Northeast Pacific. Geology 30(3): 243-246.

Letham, Bryn, Andrew Martindale, Rebecca Macdonald, Eric Guiry, Jacob Jones, and Kenneth M. Ames

2016 Postglacial Relative Sea-Level History of the Prince Rupert Area, British Columbia, Canada. Quaternary Science Reviews 153:156-191.

Letham, Bryn, Andrew Martindale, Duncan McLaren, Thomas Brown, Kenneth M. Ames, David J. W. Archer, and Susan Marsden

2015 Holocene Settlement History of the Dundas Islands Archipelago, Northern British Columbia. BC Studies 187:51-85.

Letham, Bryn, Andrew Martindale, Kisha Supernant, Thomas J. Brown, Jerome S. Cybulski, and Kenneth M. Ames

2017 Assessing the Scale and Pace of Large Shell-Bearing Site Occupation in the Prince Rupert Harbour Area, British Columbia. Journal of Island and Coastal Archaeology. DOI:10.1080/15564894.2017.1387621, accessed November 1, 2017.

Letham, Bryn, Andrew Martindale, Nicholas Waber, and Kenneth M. Ames

2018 Archaeological Survey of Dynamic Coastal Landscapes and Paleoshorelines: Locating Early Holocene Sites in the Prince Rupert Harbour Area, British Columbia, Canada. Journal of Field Archaeology 43(3):181-199.

Lepofsky, Dana, Nicole F. Smith, Nathan Cardinal, John Harper, Mary Morris, Randy Bouchard, Dorothy I. D. Kennedy, Anne K. Salomon, Michelle Puckett, and Kirsten Rowell

2015 Ancient Shellfish Mariculture on the Northwest Coast of North America. American Antiquity 80:236-259.

\section{McKechnie, Iain}

2015 Indigenous Oral History and Settlement Archaeology in Barkley Sound, Western Vancouver Island. BC Studies 187:191-225.

McKechnie, Iain, and Morely Eldridge

2013 Re-calibrating Archaeological Chronologies on the Northern Northwest Coast: Radiocarbon Data from Prince Rupert Harbour. In An Archaeology of Food and Settlement on the Northwest Coast, by Iain McKechnie, pp. 91-143. PhD dissertation, Department of Anthropology, University of British Columbia, Vancouver, Canada.

McLaren, Duncan

2008 Sea Level Change and Archaeological Site Locations on the Dundas Island Archipelago of North Coastal British Columbia. PhD dissertation, Department of Interdisciplinary Studies, University of Victoria, Victoria, British Columbia, Canada.

McLaren, Duncan, Andrew Martindale, Daryl Fedje, and Quentin Mackie

2011 Relict Shorelines and Shell Middens of the Dundas Island Archipelago. Canadian Journal of Archaeology 116:86-116.

McNeely, Roger, Arthur S. Dyke, and John R. Southon

2006 Geological Survey of Canada Open File 5049 Canadian Marine Reservoir Ages Preliminary Data Assessment. Geological Survey of Canada. DOI:10.13140/ 2.1.1461.6649, accessed March 3, 2016. 
Mangerud, Jan, and Steinar Gulliksen

1975 Apparent Radiocarbon Ages of Recent Marine Shells from Norway, Spitsbergen, and Arctic Canada. Quaternary Research 5(2):263-273.

Martindale, Andrew, Bryn Letham, Duncan McLaren, David Archer, Meghan Burchell, and Bernd R. Schone 2009 Mapping of Subsurface Shell Midden Components through Percussion Coring: Examples from the Dundas Islands. Journal of Archaeological Science 36(7):15651575 .

Martindale, Andrew, Bryn Letham, Kisha Supernant, Thomas J. Brown, Kevan Edinborough, Jonathan Duelks, and Kenneth M. Ames

2017 Urbanism in Northern Tsimshian Archaeology. Hunter Gatherer Research 3(1):133-163.

Martindale, Andrew, Susan Marsden, Katherine Patton, Angela Ruggles, Bryn Letham, Kisha Supernant, David Archer, Duncan McLaren, and Kenneth M. Ames

2017 The Role of Small Villages in Northern Tsimshian Territory from Oral and Archaeological Records. Journal of Social Archaeology 17(3):285-325.

Misarti, Nicole, Bruce Finney, Herbert D. G. Maschner, and Matthew J. Wooller

2009 Changes in Northeast Pacific Marine Ecosystems over the Last 4500 Years: Evidence from Stable Isotope Analysis of Bone Collagen from Archeological Middens. Holocene 19(8):1139-1151.

Moss, Madonna L.

1989 The Antiquity of Tlingit Settlement on Admirality Island, Southeast Alaska. PhD dissertation, Department of Anthropology, University of California, Santa Barbara.

Moss, Madonna L., and Jon M. Erlandson

1992 Forts, Refuge Rocks, and Defensive Sites: The Antiquity of Warfare along the North Pacific Coast of North America. Arctic Anthropology 29(2):73-90.

Neudorf, Christina M., Nicole Smith, Dana Lepofsky, Ginevra Toniello, and Olav B. Lian

2017 Between a Rock and a Soft Place: Using Optical Ages to Date Ancient Clam Gardens on the Pacific Northwest. PLoS ONE 12(2): e0171775. DOI:10.1371/ journal.pone.0171775, accessed December 19, 2017.

Petchey, Fiona, Atholl Anderson, Albert Zondervan, Sean Ulm, and Alan Hogg

2008 New Marine $\Delta \mathrm{R}$ Values for the South Pacific Subtropical Gyre Region. Radiocarbon 50(3):373-397.

Pluckhahn, Thomas J., Alexander D. Hodson, W. Jack Rink, Victor D. Thompson, Robert R. Hendricks, Glen Doran, Grayal Farr, Alexander Cherkinsky, and Sean P. Norman

2015 Radiocarbon and Luminescence Age Determinations on Mounds at Crystal River and Roberts Island, Florida, USA. Geoarchaeology 30(3):238-260.

Reimer, Paula J., Mike G. L. Baillie, Edouard Bard, Alex Bayliss, J. Warren Beck, Chanda J. H. Bertrand, Paul G. Blackwell, Caitlin E. Buck, George S. Burr, Kristen B. Cutler, Paul E. Damon, R. Lawrence Edwards, Richard G. Fairbanks, Michael Friedrich, Thomas P. Guilderson, Alan G. Hogg, Konrad A. Hughen, Bernd Kromer, Gerry McCormac, Sturt Manning, C. Bronk Ramsey, Ron W. Reimer, Sabine Remmele, John R. Southon, Minze Stuiver, Sahra Talamo, Frederick W. Taylor, Johannes van der Plicht, and Constanze E. Weyhenmeyer 2004 IntCal04 Terrestrial Radiocarbon Age Calibration 0-26 cal kyr BP. Radiocarbon 46(3):1029-1058.

Reimer, Paula J., Edouard Bard, Alex Bayliss, J. Warren Beck, Paul G. Blackwell, Christopher Bronk Ramsey,
Caitlin E. Buck, Hai Cheng, R. Lawrence Edwards, and Michael Friedrich

2013 IntCal13 and Marine13 Radiocarbon Age Calibration Curves 0-50,000 Years cal BP. Radiocarbon 55 (4):1869-1887.

Reimer, Ron W., and Paula J. Reimer

2017 An Online Application for $\Delta \mathrm{R}$ Calculation. Radiocarbon 59(5):1623-1627.

Rick, Torben C., Gregory A. Henkes, Darrin L. Lowery, Steven M. Colman, and Brendan J. Culleton

2012 Marine Radiocarbon Reservoir Corrections $(\Delta R)$ for Chesapeake Bay and the Middle Atlantic Coast of North America. Quaternary Research 77(1):205-210.

Rick, Torben C., René L. Vellanoweth, and Jon M. Erlandson 2005 Radiocarbon Dating and the "Old Shell" Problem: Direct Dating of Artifacts and Cultural Chronologies in Coastal and Other Aquatic Regions. Journal of Archaeological Science 32(11):1641-1648.

Robinson, Stephen W., and Gail Thompson

1981 Radiocarbon Corrections for Marine Shell Dates with Application to Southern Pacific Northwest Coast Prehistory. Syesis 14:45-57.

Russell, N., G. T. Cook, P. Ascough, and A. J. Dugmore

2010 Spatial Variation in the Marine Radiocarbon Reservoir Effect throughout the Scottish Post-Roman to Late Medieval Period: North Sea Values (500-1350 BP). Radiocarbon 52(2-3):1166-1181.

Russell, N., G. T. Cook, P. Ascough, E. M. Scott, and A. J. Dugmore

2011 Examining the Inherent Variability in $\Delta R$ : New Methods of Presenting $\Delta \mathrm{R}$ Values and Implications for MRE Studies. Radiocarbon 53(2):277-288.

Schwarcz, Henry P., Brian S. Chisholm, and Meghan Burchell

2014 Isotopic Studies of the Diet of the People of the Coast of British Columbia. American Journal of Physical Anthropology 155(3):460-468.

Shugar, Dan H., Ian J. Walker, Olav B. Lian, Jordan B. R. Eamer, Christina Neudorf, Duncan McLaren, and Daryl Fedje

2014 Post-glacial Sea-Level Change along the Pacific Coast of North America. Quaternary Science Reviews 97:170-192.

Southon, John, and Daryl Fedje

2003 A Post-glacial Record of ${ }^{14} \mathrm{C}$ Reservoir Ages for the British Columbia Coast. Canadian Journal of Archaeology 27(1):95-111.

Southon, John R., D. Erie Nelson, and John S. Vogel

1990 A Record of Past Ocean-Atmosphere Radiocarbon Differences from the Northeast Pacific. Paleoceanography 5(2):197-206.

Spzak, Paul, Michael Buckley, Christyann M. Darwent, and Michael P. Richards

2018 Long-Term Ecological Changes in Marine Mammals Driven by Recent Warming in Northwestern Alaska. Global Change Biology 24(1):490-503.

Stewart, Kathlyn M., Frances I. Stewart, and Gary Coupland 2009 Boardwalk, Northern Northwest Coast, Canada-A New Face to an Old Site. Canadian Journal of Archaeology 33(2):205-233.

Stocker, Thomas F., and Daniel G. Wright

1998 The Effect of a Succession of Ocean Ventilation Changes on ${ }^{14}$ C. Radiocarbon 40(1):359-366.

Stuiver, Minze, and Thomas F. Braziunas

1993 Modeling Atmospheric ${ }^{14} \mathrm{C}$ Influences and ${ }^{14} \mathrm{C}$ Ages of Marine Samples to 10,000 BC. Radiocarbon 35(1): 137-189. 
Stuiver, Minze, Gordon W. Pearson, and Tom Braziunas 1986 Radiocarbon Age Calibration of Marine Samples Back to 9000 cal yr BP. Radiocarbon 28(2B):980-1021. Stuiver, Minze, and Henry A. Polach

1977 Discussion of Reporting of ${ }^{14} \mathrm{C}$ Data. Radiocarbon 19(3):355-363.

Stuiver, Minze, Paula J. Reimer, and Thomas F. Braziunas 1998 High-Precision Radiocarbon Age Calibration for Terrestrial and Marine Samples. Radiocarbon 40 (3):1127-1151.

Taylor, R. E., John Southon, and Matthew R. Des Lauriers 2007 Holocene Marine Reservoir Time Series $\Delta \mathrm{R}$ Values from Cedros Island, Baja California. Radiocarbon 49 (2):899-904.

Thomson, Richard E.

1981 Oceanography of the British Columbia Coast. Canadian Special Publication of Fisheries and Aquatic Sciences No. 56. Department of Fisheries and Oceans, Ottawa, Ontario, Canada.

Voelker, Antje H. L., Michael Sarnthein, Pieter M. Grootes, Helmut Erlenkeuser, Carlo Laj, Alain Mazaud, Marie-Josée Nadeau, and Markus Schleicher

1998 Correlation of Marine ${ }^{14} \mathrm{C}$ Ages from the Nordic Seas with the GISP2 Isotope Record: Implications for
${ }^{14} \mathrm{C}$ Calibration beyond $25 \mathrm{ka}$ BP. Radiocarbon 40(1): $517-534$.

Ward, Graeme K., and Susan R. Wilson

1978 Procedures for Comparing and Combining Radiocarbon Age Determinations: A Critique. Archaeometry 20(1):19-31.

Weisler, Marshall I., Quan Hua, and Jian-xin Zhao

2009 Late Holocene ${ }^{14} \mathrm{C}$ Marine Reservoir Corrections for Hawai'i Derived from U-Series Dated Archaeological Coral. Radiocarbon 51(3):955-968.

Weisler, Marshall I., Quan Hua, Jian-xin Zhao, Ai Duc Nguyen, Luke Nothdurft, Hiroya Yamano, and Morana Mihaljević 2017 Marine Reservoir Correction for the Southern Marshall Islands for the Past 2500 Years. Radiocarbon 60(1):1-16. DOI:10.1017/RDC.2017.63, accessed December 15, 2017.

Wilson, Susan R., and Graeme K. Ward

1981 Evaluation and Clustering of Radiocarbon Age Determinations: Procedures and Paradigms. Archaeometry 23(1):19-39.

Submitted February 8, 2018; Revised April 30, 2018;

Accepted May 7, 2018 